TRANSACTIONS OF THE

AMERICAN MATHEMATICAL SOCIETY

Volume 174, December 1972

\title{
REAL LENGTH FUNCTIONS IN GROUPS
}

\author{
BY
}

\author{
NANCY HARRISON( ${ }^{1}$ )
}

ABSTRACT. This paper is a study of the structure of a group $G$ equipped with a 'length' function from $G$ to the nonnegative real numbers. The properties that we require this function to satisfy are derived from Lyndon's work on groups with integer-valued functions. A real length function is a function which assigns to each $g \in G$ a nonnegative real number $|g|$ such that the following axioms are satisfied:

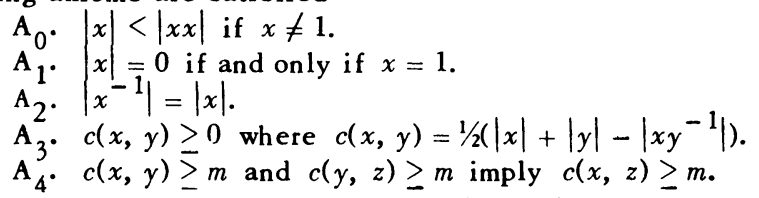

In this paper structure theorems are obtained for the cases when $G$ is abelian and when $G$ can be generated by two elements. We first prove that if $G$ is abelian, then $G$ is isomorphic to a subgroup of the additive group of the real numbers. Then we introduce a reduction process based on a generalized notion of Nielsen transformation. We apply this reduction process to finite sets of elements of $G$. We prove that if $G$ can be generated by two elements, then $G$ is either free or abelian.

1. Introduction. Lyndon [1] did some work on isolating certain cancellation arguments used in Nielsen's proof of the subgroup theorem for free groups. From this work he developed a set of properties for a 'length' function from a group to the nonnegative integers that are necessary and sufficient for any group with such a function to be a free group. The question then arises of what can be said about groups with length functions whose ranges are more general than the integers. This paper discusses groups $G$ which are equipped with a length function into the nonnegative real numbers. Structure the orems for $G$ are obtained for the cases when $G$ is abelian and when $G$ can be gene rated by two elements.

Let $G$ be an arbitrary group with a function which assigns to each $x \in G$ a nonnegative real number $|x|$. Define $c(x, y)=1 / 2(|x|+|y|-|x \bar{y}|)$ where $\bar{y}$ means $y^{-1}$. Intuitively speaking this function $c$ from $G \times G$ to the reals measures the amount of agreement on the right-hand ends of $x$ and $y$. We require that the

Received by the editors November 12, 1970.

AMS (MOS) subject classifications (1970). Primary 20E99, $20 \mathrm{~F} 15$.

Key words and phrases. Nielsen transformation, groups with functions to reals.

(1) This paper is a portion of a doctoral dissertation written at the University of Michigan. The author expresses her gratitude to Professor Roger C. Lyndon for his guidance and encouragement. Also she expresses appreciation to the University of Missouri for financial support for the preparation of this manuscript. 
following axioms be satisfied:

$A_{0} \cdot|x|<\left|x^{2}\right|$ if $x \neq 1$.

$A_{1} \cdot|x|=0$ if and only if $x=1$.

$A_{2} \cdot|\bar{x}|=|x|$.

$A_{3} \cdot c(x, y) \geq 0$.

$A_{4} . \quad c(x, y) \geq m$ and $c(x, z) \geq m$ imply $c(y, z) \geq m$.

Before proceeding we will discuss briefly some examples of groups with real length functions.

Proposition 1.1. Absolute value is a length function on the additive group of real numbers $R$.

Proof. The only property that requires any work to verify is $A_{4}$. Let $x, y$, and $z$ be real numbers such that

(1) $d(x, y) \geq m$ and

(2) $d(x, z) \geq m$.

If the signs of $x$ and $y$ or the signs of $x$ and $z$ are opposite one another, then $m=0$ and the result follows by $A_{3}$. We may then assume that $x, y$, and $z$ all have the same sign. Since both cases are very similar, we only present the details for the case in which their signs are all positive. Note that when $a$ and $b$ are both positive real numbers $c(a, b)^{\prime}=\operatorname{minimum}\{a, b\}$. Suppose first that minimum $\{y, z\} \leq x$. Then (1) implies $y \geq m$ and (2) implies $z \geq m$. If $y \leq z$ then $c(y, z)=y$ and if $z<y$ then $c(y, z)=z$. Secondly suppose $x \leq \operatorname{minimum}\{y, z\}$. Then (1) implies $x \geq m$. Therefore $c(y, z)=\operatorname{minimum}\{y, z\} \geq x \geq m$.

Proposition 1.2. Let $G=G_{1} * G_{2} * \cdots * G_{n}$ where each $G_{i}$ is a group with a real length function || . For $x$ in $G$ define $\|x\|=\sum_{i=1}^{k}\left|y_{i}\right|$ where $x=y_{1} y_{2}$ $\cdots y_{k}$ is in normal form. This is a length function for $G$.

Proof. For $x \in G$ write $x$ in the form $a x^{\prime} \bar{a}$ where $a, x^{\prime} \in G$ and $y_{1} y_{2} \cdots y_{m}$, the normal form of $x^{\prime}$, is such that either $y_{1}$ and $y_{m}$ are from different factors or else they are from the same factor and $c\left(y_{m}, \bar{y}_{1}\right)=0$. From writing $x$ in this form it is easy to see that $A_{0}$ holds. Axioms $A_{1}$ and $A_{2}$ are obvious. If $b$ and $c$ both lie in $G_{k}$, then $|b c| \leq|b|+|c|$ by $A_{3}$ in $G_{k}$. From this it follows that $A_{3}$ is $t$ rue in $G$. It remains to verify $A_{4}$. For this proof $(r, k)$ is used to denote the element $r$ from $G_{k}$.

Let

$$
\begin{aligned}
& x=\left(r_{\alpha}, i_{\alpha}\right)\left(r_{\alpha-1}, i_{\alpha-1}\right) \ldots\left(r_{2}, i_{2}\right)\left(r_{1}, i_{1}\right), \\
& \bar{y}=\left(s_{1}, j_{1}\right)\left(s_{2}, j_{2}\right) \ldots\left(s_{\beta}, j_{\beta}\right), \\
& \bar{z}=\left(t_{1}, k_{1}\right)\left(t_{2}, k_{2}\right) \ldots\left(t_{\gamma}, k_{\gamma}\right) .
\end{aligned}
$$


Let $p_{1}$ be such that $r_{q}=\bar{s}_{q}$ and $i_{q}=j_{q}$ for $q<p_{1}$ but either $r_{p_{1}} \neq \bar{s}_{p_{1}}$ or $i_{p_{1}}$ $\neq j_{p_{1}}$. Let $p_{2}$ be such that $r_{q}=\bar{t}_{q}$ and $i_{q}=k_{q}$ for $q<p_{2}$ but either $r_{p_{2}} \neq \bar{t}_{p_{2}}$ or $i_{p_{2}} \neq k_{p_{2}}$. Without loss of generality assume that $p_{1} \leq p_{2}$. We show that $c(y, z) \geq \operatorname{minimum}\{c(x, y), c(x, z)\}$ which suffices to establish $A_{4}$.

First suppose that $i_{p_{1}} \neq j_{p_{1}}$. Then $c(x, y)=\Sigma_{i=1}^{p_{1}-1}\left|r_{i}\right|$. Now $r_{q}=\bar{s}_{q}$ for $q<p_{1}$ and $r_{q}=\bar{t}_{q}$ for $q<p_{2}$. Since $p_{1} \leq p_{2}, \bar{s}_{q}=\bar{t}_{q}$ for $q<p_{1}$. It follows that $c(y, z) \geq \sum_{i=1}^{p p^{-1}}\left|s_{i}\right|=\sum_{i=1}^{p 1^{-1}}\left|r_{i}\right|=c(x, y)$.

Secondly suppose $i_{p_{1}}=j_{p_{1}}$. Then $c(x, y)=\Sigma_{i=1}^{p_{1}-1}\left|r_{i}\right|+c\left(r_{p_{1}}, \bar{s}_{p_{1}}\right)$. If $p_{1}<p_{2}$, then $r_{p_{1}}=\bar{t}_{p_{1}}$ but $r_{p_{1}} \neq \bar{s}_{p_{1}}$. It follows that $c(y, z)=\sum_{i=1}^{p_{1}-1}\left|s_{i}\right|+$ $c\left(\bar{s}_{p_{1}}, \bar{t}_{p_{1}}\right)=\Sigma_{i=1}^{p_{1}-1}\left|r_{i}\right|+c\left(\bar{s}_{p_{1}}, r_{p_{1}}\right)=c(x, y)$. We turn now to the case when $p_{1}$ $=p_{2}$. If $i_{p_{2}} \neq k_{p_{2}}$, then $c(x, z)=\sum_{i=1}^{p_{2}-1}\left|r_{i}\right|=\sum_{i=1}^{p_{1}-1}\left|s_{i}\right|=c(y, z)$. Finally suppose $i_{p_{2}}=k_{p_{2}}$ in which case $c(x, z)=\sum_{i=1}^{p_{2}-1}\left|r_{i}\right|+c\left(r_{p_{2}}, \bar{t}_{p_{2}}\right)$. Axioms $A_{2}$ and $A_{3}$ imply (see Proposition 2.1) that for $a, b \in G, c(a, b) \leq \operatorname{minimum}\{|a|,|b|\}$. By this and the fact that $j_{p_{1}}=k_{p_{1}}, c(y, z) \geq \Sigma_{i=1}^{p_{1}-1}\left|s_{i}\right|+c\left(\bar{s}_{p_{1}}, \bar{t}_{p_{1}}\right)=$ $\sum_{i=1}^{p_{1}-1}\left|r_{i}\right|+c\left(\bar{s}_{p_{1}}, \bar{t}_{p_{1}}\right)$. Now $c\left(\bar{s}_{p_{1}}, \bar{t}_{p_{1}}\right) \geq \operatorname{minimum}\left\{c\left(r_{p_{1}}, \bar{s}_{p_{1}}\right), c\left(r_{p_{1}}, \bar{t}_{p_{1}}\right)\right\}$ by $A_{4}$ in $G_{p_{1}}$. Therefore $c(y, z) \geq \operatorname{minimum}\{c(x, y), c(x, z)\}$. This completes the verification that the free product of groups with length functions has a natural length function.

Let $G$ be a group with a length function. In $\$ 2$ we present some consequences of the axioms. In the third section we prove that if $G$ is abelian, then $G$ is isomorphic to a subgroup of the additive group of real numbers. In the fourth section we introduce a reduction process for finite sets of elements from $G$. And the structure of $G$ generated by two elements is determined in $\$ 5$; such a $G$ is either free or abelian.

2. Consequences of the axioms. We want to derive some consequences of the axioms for a length function. First we consider an alternative statement of $A_{4}$

$$
c(x, y)<c(x, z) \text { implies that } c(x, y)=c(y, z) \text {. }
$$

First assume the original form of $\mathrm{A}_{4}$ and that $c(x, y)<c(x, z)$. Then $c(y, z) \geq$ $c(x, y)$ by $A_{4}$. If $c(y, z)=c(x, y)$, we are done. Suppose on the contrary that $c(y, z)>c(x, y)$. By $A_{4}, c(x, y) \geq \operatorname{minimum}\{c(x, z), c(y, z)\}>c(x, y)$, a contradiction. Conversely assume the new statement, which for now we shall call $\mathrm{A}^{\prime}{ }_{4}$, and that minimum $\{c(x, y), c(x, z)\} \geq m$. If $c(y, z) \geq c(x, y)$, we are done. And if $c(y, z)<c(x, y)$, then $A^{\prime}{ }_{4}$ implies $c(y, z)=c(x, z) \geq m$. Therefore the two statements $A_{4}$ and $A_{4}^{\prime}$ are equivalent.

We now state some immediate consequences of the axioms that we will be using throughout. 
Proposition 2.1. $c(x, \bar{y}) \leq \operatorname{minimum}\{|x|,|y|\}$.

Proof. By symmetry it suffices to show the result for $|x| \geq|y|$. Axiom $A_{3}$ implies that $c(x y, y) \geq 0$. It follows by $A_{2}$ that

$$
c(x, \bar{y})=1 / 2(|x|+|\bar{y}|-|x y|) \leq 1 / 2(|x|+|\bar{y}|+|y|-|x|)=|y| .
$$

Proposition 2.2. $c(x y, y)=|y|-c(x, \bar{y})$ [1, Proposition 2.1, p. 210].

Proposition 2.3. $c(x, \bar{y})+c(y, \bar{z}) \geq|y|$ implies that $|x y z| \leq|x|-|y|+|z|$ [1, Proposition 2.2, p. 211].

Proposition 2.4. $c(x, \bar{y})+c(y, \bar{z})<|y|$ implies that $c(x y, \bar{z})=c(y, \bar{z})[1$, Proposition 2.3, p. 211].

Proposition 2.5. If $|x|=|y|$ and $c(x, y)+c(\bar{x}, \bar{y}) \geq|x|$, then $x=y[1$, Proposition 2.4, p. 211].

Proposition 2.6. If $d=c(x, \bar{x})$, then $\left|x^{n+1}\right|=|x|+n(|x|-2 d)$ where $n$ is an arbitrary nonnegative integer [1, Proposition 2.5, p. 212].

It follows from this proposition that the lengths $\left|x^{n}\right|$ are unbounded. If $x$ $\epsilon G$ such that $c(x, \bar{x})=0$, we say that $x$ is cyclically reduced. For cyclically reduced $x,\left|x^{n}\right|=n|x|$.

Corollary 2.7. Every $x \in G, x \neq 1$, bas infinite order.

Proof. Since $x \neq 1,|x|>0$ by $A_{1}$. If $x$ has finite order, say $n$, then $\left|x^{n}\right|$ $=0$ by $A_{1}$. But then $\left|x^{n}\right|<|x|$, contradicting Proposition 2.6. Hence the order of $x$ is infinite.

Finally we need to establish the fact that the function $f(x)=\left|x^{2}\right|-|x|$ from $G$ to the nonnegative reals is a class function. The proof is long and requires a number of case distinctions, but it is not difficult. We remark that this is a key factor in one case of the determination of the structure of a group $G$ generated by a pair of elements.

In this proof and most of those following, $A_{2}$ and its immediate consequence, $c(x, y)=c(y, x)$, are used frequently. Since constant referral is burdensome, for the remainder of the paper we will use them without specific mention.

Theorem 2.8. For arbitrary $g, x \in G,\left|x^{2}\right|-|x|=\left|\bar{g} x^{2} g\right|-|\bar{g} x g|$.

Proof. If $g$ or $x$ is 1 , the equality is trivial. Henceforth assume that $g \neq$ 1 and $x \neq 1$. Consider $c(\bar{g}, \bar{x})$ and $c(\bar{g}, x)$.

Case 1. $c(\bar{g}, \bar{x})=0$ and $c(\bar{g}, x)=0$.

The se equations are equivalent to

$$
|\bar{g} x|=|g|+|x|=|x g| \text {. }
$$


If $c(\bar{g} x, \bar{g}) \neq 0$, then $c(\bar{g}, x)<c(\bar{g}, \bar{g} x)$. Axiom $A_{4}$ then implies $c(x, \bar{g} x)=0$ so $|\bar{g} x|=|g|-|x|$. But this contradicts (1) since $|x|>0$ by $A_{1}$. Therefore $c(\bar{g} x, \bar{g})$ $=0$ and

$\left(i_{1}\right)$

$$
|\bar{g} x g|=2|g|+|x| .
$$

If $c\left(\bar{g}, \bar{x}^{2}\right) \neq 0$, then $c(\bar{g}, \bar{x})<c\left(\bar{g}, \bar{x}^{2}\right)$. Axiom $A_{4}$ implies $c\left(\bar{x}, \bar{x}^{2}\right)=0$, hence $\left|x^{2}\right|=0$. But this contradicts $A_{0}$ since $|x|>0$ by $A_{1}$. Therefore $c\left(\bar{g}, \bar{x}^{2}\right)=0$ so

$$
\left|\bar{g} x^{2}\right|=|g|+\left|x^{2}\right| \text {. }
$$

If $c\left(\bar{g} x^{2}, \bar{g}\right) \neq 0$, then $c(\bar{g}, x)<c\left(\bar{g}, \bar{g} x^{2}\right)$; so $c\left(x, \bar{g} x^{2}\right)=0$ by $A_{4}$. Using (ii ${ }_{1}$ ) and (1) it follows that $\left|x^{2}\right|=0$, a contradiction of $A_{0}$ since $|x|>0$ by $A_{1}$. Therefore $c\left(\bar{g} x^{2}, \bar{g}\right)=0$, and using (ii $\left.{ }_{1}\right)$

$$
\left|\bar{g} x^{2} g\right|=2|g|+\left|x^{2}\right|
$$

Then $\left(i_{1}\right)$ and $\left(\mathrm{iii}_{1}\right)$ imply

$$
\left|\bar{g} x^{2} g\right|-|\bar{g} x g|=\left|x^{2}\right|-|x| .
$$

Case 2. $c(\bar{g}, \bar{x}) \neq 0$ and $c(\bar{g}, x)=0$.

Then

$$
|\bar{g} \bar{x}|=|g|+|x| \neq|\bar{g} x| \text {. }
$$

Since $c(\bar{g}, x)<c(\bar{g}, \bar{x}), A_{4}$ implies $c(x, \bar{x})=0$ and

$$
\left|x^{2}\right|-|x|=|x| \text {. }
$$

Consider $c(\bar{g} x, \bar{g})$. We will treat the zero and nonzero cases separately.

First assume $c(\bar{g} x, \bar{g})=0$, so

$$
|\bar{g} x g|=|\bar{g} x|+|g| \text {. }
$$

Now $c(\bar{g}, \bar{g} x)=0<c(\bar{g}, \bar{x})$ implies by $A_{4}$ that $c(\bar{g} x, \bar{x})=0$, so $\left(\mathrm{i}_{2 \mathrm{a}}\right)$

$$
\left|\bar{g} x^{2}\right|=|\bar{g} x|+|x| \text {. }
$$

Consider $c\left(\bar{g} x^{2}, \bar{g}\right)$. First assume $c\left(\bar{g} x^{2}, \bar{g}\right)=0$. It follows from $\left(\mathrm{i}_{2 \mathrm{a}}\right)$ and $(2 \mathrm{a})$ that $\left|\bar{g} x^{2} g\right|-|\bar{g} x g|=|x|$. Since $\left|x^{2}\right|-|x|=|x|$ by $\left(*_{2}\right)$, this is our desired result. Next assume $c\left(\bar{g}, \bar{g} x^{2}\right) \neq 0$. It is easy to see that $c(\bar{g} x, \bar{g} \bar{x})=0$. If, on the contrary, $c(\bar{g} x, \bar{g} \bar{x})>0=c(\bar{g}, \bar{g} x), A_{4}$ implies $c(\bar{g}, \bar{g} \bar{x})=0$. From this $|\bar{g} x g|$ $=|g|+|\bar{g} \bar{x}|$, but from (2a), $|\bar{g} x g|=|\bar{g} x|+|g|$. Since $|\bar{g} \bar{x}| \neq|\bar{g} x|$ by (2), we have a contradiction. From $c(\bar{g} x, \bar{g} \bar{x})=0,(2)$, and $(2 a),\left|\bar{g} x^{2} g\right|-|\bar{g} x g|=|x|$. In view of $\left(*_{2}\right)$, this is our desired result.

Secondly assume $c(\bar{g} x, \bar{g}) \neq 0$. Since $c(\bar{g}, x)=0<c(\bar{g}, \bar{g} x), c(x, \bar{g} x)=0$ by $A_{4}$. Hence 


$$
\left(i_{2 b}\right) \quad|\bar{g} x|=|g|-|x| .
$$

It is easy to see that $c(\bar{g} x, \bar{g})=c(\bar{g} x, \bar{g} \bar{x})$. We assume the contrary and derive a contradiction. If $c(\bar{g} x, \bar{g})<c(\bar{g} x, \bar{g} \bar{x})$, then $A_{4}$ implies $c(\bar{g} x, \bar{g})=c(\bar{g}, \bar{g} \bar{x})$. It follows that $|\bar{g} x|=|\bar{g} \bar{x}|$, but this contradicts (2). If $c(\bar{g} x, \bar{g} \bar{x})<c(\bar{g} x, \bar{g})$, then $A_{4}$ implies $c(\bar{g} x, \bar{g} \bar{x})=c(\bar{g} \bar{x}, \bar{g})$, hence $|\bar{g} x|-\left|\bar{g} x^{2} g\right|=|g|-|\bar{g} \bar{x} g|$. Using (i $\left.{ }_{2 b}\right)$ it follows that $\left|\bar{g} x^{2} g\right|-|\bar{g} x g|=-|x|$. But this contradicts $A_{0}$. Therefore $c(\bar{g} x, \bar{g})=c(\bar{g} x, \bar{g} \bar{x})$. Using (2), $\left|\bar{g} x^{2} g\right|-|\bar{g} x g|=|x|$ which in view of $\left(*_{2}\right)$ is our desired result.

Case 3. $c(\bar{g}, \bar{x})=0$ and $c(\bar{g}, x) \neq 0$.

This follows immediately from Case 2 .

Case 4. $c(\bar{g}, \bar{x}) \neq 0$ and $c(\bar{g}, x) \neq 0$.

We will treat separately the three possiblilites that arise in comparing $c(\bar{g}, \bar{x})$ and $c(\bar{g}, x)$.

(4a) $c(x, \bar{g})=c(\bar{x}, \bar{g})$.

Axiom $A_{4}$ implies $c(x, \bar{x}) \geq c(x, \bar{g})$ so $1 / 2|x|>c(x, \bar{g})$. Then $c(x, \bar{x})+c(x, \bar{g})<$ $|x|$ implying by Proposition 2.4 that $c\left(x^{2}, \bar{g}\right)=c(x, \bar{g})$. Thus

$$
\left|x^{2}\right|-|x|=\left|x^{2} g\right|-|x g| \text {. }
$$

Also $c(\bar{g}, \bar{x})+c(x, \bar{g})<|x|$ so Proposition 2.4 implies $c(\bar{g} x, \bar{g})=c(x, \bar{g})$. Thus $\left(\mathrm{i}_{4 \mathrm{a}}\right)$

$$
|\bar{g} x g|=2|x g|-|x| \text {. }
$$

Next we need to examine $\left|\bar{g} x^{2} g\right|$; we claim that $c\left(\bar{g}, \overline{x^{2} g}\right)=c(\bar{g}, x)$. Since $c(\bar{g}, x)<1 / 2|x|, c(\bar{g}, x)+c(\bar{x}, x)<|x|$, so Proposition 2.4 implies $c(\bar{g} \bar{x}, x)=$ $c(\bar{x}, x)$. It follows that $c(\bar{g} \bar{x}, x)+c(x, \bar{g})<|x|$; Proposition 2.4 then implies $c\left(\bar{g} \bar{x}^{2}, \bar{g}\right)=c(x, \bar{g})$. Thus

$$
\text { (ii } \left._{4 \mathrm{a}}\right) \quad\left|\bar{g} \bar{x}^{2} g\right|=\left|x^{2} g\right|-|x|+|x g| \text {. }
$$

Using ( $\mathrm{i}_{4 \mathrm{a}}$ ) we obtain $\left|\bar{g} \bar{x}^{2} g\right|-|\bar{g} x g|=\left|x^{2} g\right|-|x g|$. In view of $\left(*_{4 \mathrm{a}}\right)$ this is our desired result.

(4b) $c(\bar{g}, \bar{x})<c(\bar{g}, x)$.

Axiom $A_{4}$ implies $c(\bar{g}, \bar{x})=c(\bar{x}, x)$; hence

$$
\left|x^{2}\right|-|x|=|\bar{g} x|-|g| \text {. }
$$

Since $c(\bar{g}, \bar{x})<c(\bar{g}, x),|\bar{g} \bar{x}|<|\bar{g} x|$ and $c(\bar{g} \bar{x}, \bar{g})<c(\bar{g} x, \bar{g})$. Applying $A_{4}$ to this we obtain $c(\bar{g} \bar{x}, \bar{g})=c(\bar{g} \bar{x}, \bar{g} x)$. Therefore $\left|\bar{g} x^{2} g\right|-|\bar{g} x g|=|\bar{g} x|-|g|$, which in view of $\left(*_{4 b}\right)$ is our desired result.

(4c) $c(\bar{g}, x)<c(\bar{g}, \bar{x})$. 
This follows immediately from (4b).

\section{Abelian groups with real length functions.}

Introduction. Let $G$ be a nontrivial abelian group with a real length function. We prove that $G$ is isomorphic to a subgroup of the additive group of real numbers $R$.

$A$ new length function on $G$. Choose a nontrivial element $a$ of $G$ and let $d=c(a, \bar{a})$. Define a function \|\| from $G$ to $R$ as follows:

$$
\begin{aligned}
& \text { if } x \neq 1 \text {, then }\|x\|=|x|-2 d \text {; } \\
& \text { if } x=1 \text {, then }\|x\|=0 .
\end{aligned}
$$

We claim that \|\| is a length function.

Lemma 3.1. If $x$ and $y$ are nontrivial elements of $G$, then $c(x, y) \geq d$ where $d=c(a, \bar{a})$.

Proof. It suffices to show that $c(a, x) \geq d$ for $x \in G, x \neq 1$. For $c(a, x) \geq$ $d$ and $c(a, y) \geq d$ imply by $A_{4}$ that $c(x, y) \geq d$. We assume that there exists some $x \in G, x \neq 1$ such that $c(a, x)<d$ and derive a contradiction. Now $c(a, x)<$ $c(a, \bar{a})=d$ implies by $A_{4}$ that $c(a, x)=c(\bar{a}, x)$; hence $|a \bar{x}|=|\bar{a} \bar{x}|$.

First consider the case in which $\left|a^{2}\right| \leq|a \bar{x}|$. Since $|a \bar{x}|=|\bar{a} \bar{x}|$,

$$
c(a \bar{x}, \bar{a} \bar{x})=1 / 2\left(2|a \bar{x}|-\left|a^{2}\right|\right) \geq 1 / 2|a \bar{x}| \text {. }
$$

It follows that $c(a \bar{x}, \bar{a} \bar{x})+c(x \bar{a}, x a) \geq|a \bar{x}|=|\bar{a} \bar{x}|$, so Proposition 2.5 implies $a \bar{x}=\bar{a} \bar{x}$. Then $a=\bar{a}$, contradicting Corollary 2.7.

Secondly assume that $|a \bar{x}|<\left|a^{2}\right|$. Since $|a \bar{x}|=|\bar{a} \bar{x}|, c\left(a \bar{x}, a^{2}\right)=1 / 2\left|a^{2}\right|=$ $c\left(\bar{a}^{2}, \bar{a} x\right)$. Proposition 2.3 then implies that $\left|x^{2}\right| \leq|a \bar{x}|-\left|a^{2}\right|+|\bar{a} x|<\left|a^{2}\right|$. Now $c\left(a \bar{x}, \bar{x}^{2}\right)=1 / 2\left|x^{2}\right|$, so that $c\left(a \bar{x}, \bar{x}^{2}\right)<c\left(a \bar{x}, a^{2}\right)$. By $A_{4}, c\left(a \bar{x}, \bar{x}^{2}\right)=c\left(a^{2}, \bar{x}^{2}\right)$; from this it follows that $\left|a^{2}\right|=\left|a^{2} x^{2}\right|$. Since $c\left(a^{2}, a^{2} x^{2}\right)+c\left(\bar{a}^{2}, \bar{x}^{2} \bar{a}^{2}\right)=2\left|a^{2}\right|-$ $\left|x^{2}\right|>\left|a^{2}\right|$, Proposition 2.5 implies $a^{2}=a^{2} x^{2}$. This yields $x^{2}=1$, a contradiction of Corollary 2.7.

Corollary 3.2. If $x \in G, x \neq 1$, then $|x|>2 d$ where $d=c(a, \bar{a})$.

Proof. Since $c\left(x, x^{2}\right)=1 / 2\left|x^{2}\right|$ and $c(x, \bar{x})=|x|-1 / 2\left|x^{2}\right|$, Lemma 3.1 implies $|x| \geq d+1 / 2\left|x^{2}\right| \geq 2 d$. If $|x|=2 d$, then $\left|x^{2}\right|=2 d=|x|$, contradicting $A_{0}$ as $x \neq$ 1. Therefore $|x|>2 d$.

Define $\tilde{c}(x, y)=1 / 2(\|x\|+\|y\|-\|x \bar{y}\|)$. Note that if $x, y$, and $x \bar{y}$ are all nontrivial then $\tilde{c}(x, y)=c(x, y)-d$.

Lemma 3.3. $\tilde{c}(x, y) \leq \tilde{c}(x, x)$ for $x, y \in G$. 
Proof. The assertion is trivial if $x$ or $y$ is the identity, whence we may suppose that $x \neq 1$ and $y \neq 1$. Furthermore no generality is lost in assuming that $|x| \leq|y|$ since it implies $\tilde{c}(x, x) \leq \tilde{c}(y, y)$. First consider the case that $|x|<|y|$. It suffices to show that $c(x, y) \leq|x|-d$; for from this it follows that

$$
\widetilde{c}(x, y)=c(x, y)-d \leq|x|-2 d=\widetilde{c}(x, x) .
$$

We assume on the contrary that $c(x, y)>|x|-d$ which is equivalent to $|x \bar{y}|<$ $|y|-|x|+2 d$ and derive a contradiction. Since $|x|<|y|, c(x \bar{y}, x)<c(x \bar{y}, \bar{y})$ which yields by $A_{4}$ that $c(x \bar{y}, x)=c(x, \bar{y})$. From this and our assumption we conclude that $|x y|=2|y|-|x \bar{y}|>|y|+|x|-2 d$. But this is equivalent to $c(x, \bar{y})$ $<d$, a contradiction of Lemma 3.1. We turn now to the case when $|x|=|y|$. If $c(x, y)>|x|-d$, then $c(x, y)+c(\bar{x}, \bar{y})>|x|=|y|$ by Corollary 3.2. Proposition 2.5 implies that $x=y$ so $\tilde{c}(x, y)=\tilde{c}(x, x)$. If $c(x, y) \leq|x|-d$, then $\tilde{c}(x, y)=$ $c(x, y)-d \leq|x|-2 d=\tilde{c}(x, x)$.

Proposition 3.4. The function from $G$ to $R$ defined by $\|x\|=|x|-2 d$ is a length function.

Proof. $A_{0}$. If $x \neq 1$, then $\|x\|<\left\|x^{2}\right\|$. This follows immediately from $A_{0}$ for the original length function.

$A_{1} .\|x\|=0$ if and only if $x=1$. If $x=1$, then $\|x\|=0$ by definition. And if $\|x\|=0$, then $x=1$ or $|x|=2 d$. By Corollary $3.2,|x|>2 d$ unless $x=1$. Thus $x=1$.

$A_{2} \cdot\|\bar{x}\|=\|x\|$. This follows immediately from $A_{2}$ for the original length function.

$\mathrm{A}_{3} \cdot \tilde{c}(x, y) \geq 0$. If $x=1$, then $\tilde{c}(x, y)=1 / 2(\|1\|+\|y\|-\|\bar{y}\|)=0$ by $\mathrm{A}_{2}$. The case in which $y=1$ is handled similarly. We now assume that $x \neq 1$ and $y \neq 1$. If $x \neq y$, then $\tilde{c}(x, y)=c(x, y)-d \geq 0$ by Lemma 3.1. If $x=y$, then $\tilde{c}(x, x)=|x|-2 d>0$ by Corollary 3.2.

$A_{4}$. If $\tilde{c}(x, y) \geq m$ and $\tilde{c}(x, z) \geq m$, then $\tilde{c}(y, z) \geq m$. If $1 \in\{x, \dot{y}, z\}$, then $m=0$ and the result follows by $A_{3}$. If $y=z$, the result follows from Lemma 3.3. We may assume that $1 \notin\{x, y, z\}$ and $y \neq z$. If $x^{\prime}$ and $y^{\prime}$ are both nontrivial elements of $G$, then

$$
c\left(x^{\prime}, y^{\prime}\right)= \begin{cases}\tilde{c}\left(x^{\prime}, y^{\prime}\right)+d & \text { if } x^{\prime} \neq y^{\prime}, \\ \tilde{c}\left(x^{\prime}, y^{\prime}\right)+2 d & \text { if } x^{\prime}=y^{\prime} .\end{cases}
$$

Now $\tilde{c}(x, y) \geq m$ and $\tilde{c}(x, z) \geq m$ imply respectively that $c(x, y) \geq m+d$ and $c(x, z) \geq m+\bar{d}$. Axiom $\mathrm{A}_{4}$ for the original length function implies $c(y, z) \geq$ $m+d$. Since $y \neq z$, it follows that $\tilde{c}(y, z) \geq m$.

We have adjusted the original length function on $G$ so that $\tilde{c}(a, \bar{a})=0$ 
from which it follows by Proposition 2.6 that $\left\|a^{n}\right\|=n\|a\|$. Henceforth denote $\|x\|$ by $|x|$ and $\tilde{c}(x, y)$ by $c(x, y)$.

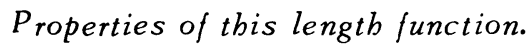

Proposition 3.5. For all $x$ in $G, c(x, \bar{x})=0$ or equivalently $\left|x^{2}\right|=2|x|$.

Proof. We assume that there exists some $x \in G, x \neq 1$ with $c(x, \bar{x}) \neq 0$ and derive a contradiction. First suppose $c(a, x) \neq 0$. Since $c(a, \bar{a})=0<c(a, x)$, $\mathrm{A}_{4}$ implies $c(x, \bar{a})=0$. And since $c(x, \bar{a})=0<c(x, \bar{x}), \mathrm{A}_{4}$ implies $c(\bar{x}, \bar{a})=0$. But this contradicts the fact that $c(a, x) \neq 0$. We now examine the case that $c(a, x)=0$. Since $c(x, \bar{x}) \neq 0, \mathrm{~A}_{4}$ yields that $c(\bar{x}, a)=0$. Thus $|a \bar{x}|=|\bar{a} \bar{x}|=$ $|a|+|x|$. Since $\left|a^{2}\right|=2|a|$, it follows that $c(a \bar{x}, \bar{a} \bar{x})+c(x a, x \bar{a})=2|x|$. If $|x|$ $\geq|a|$, then Proposition 2.5 implies that $a \bar{x}=\bar{a} \bar{x}$, contrary to Corollary 2.7. If $|a|>|x|$, then Proposition 2.4 implies $c\left(a^{2}, x \bar{a}\right)=c(x a, x \bar{a})$, whence $\left|a^{3} \bar{x}\right|=$ $3|a|-|x|$. But $c(a, x)=0<c\left(a, a^{3}\right)=|a|$ yields by $A_{4}$ that $c\left(a^{3}, x\right)=0$, whence $\left|a^{3} \bar{x}\right|=3|a|+|x|$. Since $|x|>0$ by $A_{1}$, we have a contradiction.

Proposition 3.6. For $x, y \in G,|x|=|y|$ implies $y=x$ or $y=\bar{x}$.

Proof. We will show that if $c(x, y) \neq 0$, then $y=x$ and if $c(x, y)=0$, then $y=\bar{x}$. Suppose first that $c(x, y) \neq 0$. Since $c(x, \bar{x})=0$ by Proposition 3.5, $c(\bar{x}, y)=0$ by $A_{4}$. Using our hypothesis that $|x|=|y|$ and Proposition 3.5, it follows that $c(x y, \bar{x} y)=1 / 2|\bar{x} y|=c(\overline{x y} ; \bar{x} y)$. Axiom $A_{4}$ then implies $c(x y, \bar{x} \bar{y}) \geq$ $1 / 2|\bar{x} y|$, but Proposition 3.5 implies $c(x y, \overline{x y})=0$. Thus $|\bar{x} y|=0$ so $y=x$ by $A_{1}$. Secondly suppose $c(x, y)=0$. The hypothesis and Proposition 3.5 then yield that $c(x y, \bar{x} y)=1 / 2|x y|=c(\overline{x y}, \bar{x} y)$. By $A_{4}, c(x y, \overline{x y}) \geq 1 / 2|x y|$ and by Proposition 3.5, $c(x y, \overline{x y})=0$. It follows that $|x y|=0$ so $y=\bar{x}$ by $A_{1}$.

Proposition 3.7. For all $x, y \in G$ either

(i) $c(x, \bar{y})=0$ and $c(x, y)=\operatorname{minimum}\{|x|,|y|\}$ or

(ii) $c(x, y)=0$ and $c(x, \bar{y})=$ minimum $\{|x|,|y|\}$.

Proof. Suppose $c(x, \bar{y})=0$. Then since $\left|y^{2}\right|=2|y|$ by Proposition 3.5, $c(x \bar{y}, x y)=1 / 2(|x \bar{y}|+|x|-|y|)$. If $c(x \bar{y}, x y)=0$, then $|x \bar{y}|=|y|-|x| \geq 0$, whence $c(x, y)=|x| \leq|y|$. If $c(x \bar{y}, x y) \neq 0$, then since $c(x \bar{y}, \overline{x \bar{y}})=0$ by Proposition 3.5, $\mathrm{A}_{4}$ implies $0=c(x y, \overline{x \bar{y}})$. Since $\left|x^{2}\right|=2|x|$, it follows that $c(x, y)=|y| \leq|x|$.

Suppose $c(x, \bar{y})>0$. Since $c(y, \bar{y})=0, \mathrm{~A}_{4}$ implies $c(x, y)=0$. By the above $c(x, \bar{y})=\operatorname{minimum}\{|x|,|y|\}$.

Establishment of the isomorphism. Define $\phi: G \rightarrow R$ as follows:

$$
\phi(x)= \begin{cases}+|x| & \text { if } c(a, x) \neq 0 \\ -|x| & \text { if } c(a, x)=0\end{cases}
$$


Lemma 3.8. $\phi: G \rightarrow R$ is a bomomorphism.

Proof. Let $x, y \in G$. If $x$ or $y$ is 1 , clearly $\phi(x y)=\phi(x)+\phi(y)$ since $\phi(1)$ $=0$. Assume now that $x \neq 1$ and $y \neq 1$. We first consider the case that $c(a, x y)$ $=0$, whence $\phi(x y)=-|x y|$. First suppose $\phi(x)$ and $\phi(y)$ have opposite signs; for definiteness say $\phi(x)=+|x|$ and $\phi(y)=-|y|$. Then $c(a, x y)<c(a, x)$ implies by $A_{4}$ that $c(x y, x)=0$ so $|x y|=|y|-|x|$. Hence $\phi(x y)=-|x y|=-|y|$ $+|x|=\phi(x)+\phi(y)$. Secondly suppose $\phi(x)$ and $\phi(y)$ both have positive signs. Now $0=c(a, x y)<c(a, x)$ implies by $A_{4}$ that $c(x y, x)=0$ so $|x y|=|y|-|x|$. Since $c(a, y)>0$, a similar argument shows $|x y|=|x|-|y|$. It follows that $|x|=$ $|y|$ so $x=\bar{y}$ by $A_{1}$. Then $c(a, \bar{y})$ and $c(a, y)$ both are nonzero as $\phi(x)$ and $\phi(y)$ both have positive signs. But this contradicts Proposition 3.7. Thirdly suppose $\phi(x)$ and $\phi(y)$ both have negative signs. Since $c(a, y)=0$, Proposition 3.7 implies $c(a, \bar{y}) \neq 0$. Then $0=c(a, x)<c(a, \bar{y})$ so $c(x, \bar{y})=0$ by $A_{4}$. Hence $\phi(x y)=-|x y|=-|x|-|y|=\phi(x)+\phi(y)$.

Next we examine the case that $0<c(a, x y)$. We will first show that for $z$ $\epsilon G, \phi(\bar{z})=-\phi(z)$. If $c(a, z) \neq 0$, Proposition 3.7 implies $c(a, \bar{z})=0$. Thus by definition of $\phi, \phi(\bar{z})=-|\bar{z}|=-|z|=-\phi(z)$. If $c(a, z)=0$, then Proposition 3.7 implies $c(a, \bar{z})=\operatorname{minimum}\{|a|,|z|\}$. Axiom $A_{1}$ implies that $c(a, \bar{z})>0$ or $z=1$. If $c(a, \bar{z})>0$, then by the above $\phi(\overline{\bar{z}})=-\phi(\bar{z})$, whence $\phi(\bar{z})=-\phi(z)$. And if $z=1$, the assertion is trivial. Since $c(a, x y) \neq 0$, Proposition 3.7 implies $c(a, \overline{x y})=0$. By the first case that we discussed $\phi(\bar{x} \bar{y})=\phi(\bar{x} \bar{y})=\phi(\bar{x})+\phi(\bar{y})$, and by the above $\phi(\overline{x y})=-\phi(x y)$. Thus $\phi(x y)=-\phi(\bar{x})-\phi(\bar{y})$. Another application of the fact that $\phi(\bar{z})=-\phi(z)$ yields $\phi(x y)=\phi(x)+\phi(y)$ as desired. This completes the proof that $\phi$ is a homomorphism.

Lemma 3.9. $\phi: G \rightarrow R$ is one-to-one.

Proof. Let $x \in G$ such that $\phi(x)=0$. The definition of $\phi$ forces $|x|=0$ so $x=1$ by $A_{1}$.

These last two lemmas yield the result we set out to prove.

Theorem 3.10. Let $G$ be an abelian group with a real length function. Then $G$ is isomorpbic to a subgroup of the additive group of real numbers $R$.

4. A reduction process. In this section we introduce a reduction process that we apply to finite sets of elements from a group with a real length function. We will first discuss some results that we use from Lyndon's paper [1]. Lyndon isolated some cancellation ideas from Nielsen's proof of the subgroup theorem for free groups, and he proved a theorem that a product of elements is not the identity provided there is not too much cancellation in forming the product of any 
three consecutive factors. The arguments he uses for this are valid for groups with real length functions.

Lemma 4.1. Let $x_{1}, x_{2}, \ldots, x_{n}$ be elements of a group $G$ with a real length function. If $c\left(x_{i-1}, \bar{x}_{i}\right)+c\left(x_{i}, \overline{x_{i+1}}\right)<\left|x_{i}\right|$ for all $i$ such that $1<i<n$, then

(i) $\left|x_{1} x_{2} \cdots x_{n}\right|=\sum_{i=1}^{n}\left|x_{i}\right|-2 \sum_{i=1}^{n-1} c\left(x_{i}, \overline{x_{i+1}}\right)$ and

(ii) $x_{1} x_{2} \cdots x_{n} \neq 1$ for $n \geq 3$ [1, Lemmas 6.1 and 6.2, pp. 222-223].

Theorem 4.2. Let $x_{1}, x_{2}, \cdots, x_{n}$ be nontrivial elements of a group $G$ with a real length function. Assume

(4.2a) $c\left(x_{i}, \overline{x_{i+1}}\right) \leq \operatorname{minimum}\left\{1 / 2\left|x_{i}\right|, 1 / 2\left|x_{i+1}\right|\right\}$ for $1 \leq i<n$;

(4.2b) $c\left(x_{i-1}, \bar{x}_{i}\right)=c\left(x_{i}, \bar{x}_{i+1}\right)=1 / 2\left|x_{i}\right|$ does not hold for any two consecutive factors $x_{i}$;

(4.2c) the equation of (4.2b) implies $\left|x_{i-1} x_{i} x_{i+1}\right|=\left|x_{i-1}\right|-\left|x_{i}\right|+\left|x_{i+1}\right|$.

Then the conclusion of Lemma 4.1 bolds and $x_{1} x_{2} \cdots x_{n} \neq 1$ if $n \geq 1[1$, Theorem 6.4, p. 224].

Let $G$ be an arbitrary group and $x_{1}, x_{2}, \ldots, x_{n} \in G$. The following transformations of $X=\left(x_{1}, x_{2}, \ldots, x_{n}\right)$ are called elementary Nielsen transformations:

(1) permuting the $x_{i}$;

(2) replacing $x_{i}$ by $\bar{x}_{i}$ for a single index $i$;

(3) replacing $x_{i}$ by one of $x_{i} x_{j}, x_{i} \bar{x}_{j}, x_{j} x_{i}, \bar{x}_{j} x_{i}$ for fixed $i$ and $j, i \neq j$.

If a Nielsen transformation is applied to a sequence $X$, the new sequence $X^{\prime}$ generates the same subgroup of $G$ as $X$ does.

For the remainder of this section let $G$ be a group with a real length function on it. We shall say that the right half of $x$ is isolated from $y$ if $c(x, y)<$ $1 / 2|x|$ or equivalently $|x \bar{y}|>|y|$. Similarly the left half of $x$ is isolated from $y$ if $c(\bar{y}, \bar{x})<1 / 2|x|$ or equivalently $|\bar{y} x|>|y|$. The right half of $x$ is said to be isolated in a set $X$ if the right half of $x$ is isolated from all $y$ and $\bar{y}$ where $y \in X, y \neq x, y \neq \bar{x}$. A similar definition can be made for the left half of $x$ being isolated in $X$. And we shall say that $x$ ends with the right balf of $y$ if $|y \bar{x}|=$ $|x|$ and begins with the left half of $y$ if $|\bar{y} x|=|x|$.

Definition. $X=\left\{x_{1}, x_{2}, \ldots, x_{n}\right\}$ is Nielsen reduced if for $i \neq j,\left|x_{i}^{\epsilon} x_{j}^{\delta}\right| \geq$ $\operatorname{maximum}\left\{\left|x_{i}\right|,\left|x_{j}\right|\right\}$ where $\epsilon, \delta \in\{+1,-1\}$.

Definition. $X=\left\{x_{1}, x_{2}, \ldots, x_{n}\right\}$ is fully Nielsen reduced if it is Nielsen reduced and for each $x \in X$ either $\left|x y^{\epsilon}\right|>|y|$ for all $y \in X, y \neq x$, and $\epsilon \epsilon$ $\{+1,-1\}$ or $\left|\bar{x} y^{\epsilon}\right|>|y|$ for all $y \in X, y \neq x$, and $\epsilon \in\{+1,-1\}$; that is for each $x \in X$ either its right half or its left half is isolated in $X$.

Theorem 4.3. If a set $X$ of elements from a group $G$ with a real length 
function is Nielsen reduced, then there exists a finite number of Nielsen transformations which we can apply to $X$ to obtain a set $X^{\prime}$ which is fully Nielsen reduced.

Proof. This proof is an adaption of one given in Magnus, Karrass, and Solitar [2, Theorem 3.1, pp. 126-128]. Let $X=\left\{w_{1}, w_{2}, \ldots, w_{n}\right\}$ be a Nielsen reduced set where $\left|w_{1}\right| \leq\left|w_{2}\right| \leq \cdots \leq\left|w_{n}\right|$. Assume that $X=\left\{w_{1}, \ldots, w_{t-1}\right\}$ is fully Nielsen reduced. First we modify $X$ so that the right half of every element in it is isolated. If the right half of $w_{\lambda}$ is not isolated in $\tilde{X}$, then replace $w_{\lambda}$ by $\bar{w}_{\lambda}$. In this way we obtain a set $X^{\prime}=\left\{w_{1}^{\prime}, \ldots, w_{t-1}^{\prime}\right\}$ in which $w_{i}^{\prime} \in\left\{w_{i}, \bar{w}_{i}\right\}$ and the right half of every element of $X^{\prime}$ is isolated in $X^{\prime}$.

Next we isolate the right half of each element in $X^{\prime}$ from $w_{t}$ and $\bar{w}_{t}$. If $\left|w_{\lambda}^{\prime} \bar{w}_{t}\right|>\left|w_{t}\right|$, then the right half of $w_{\lambda}^{\prime}$ is isolated from $w_{t}$ already. If $\left|w_{\lambda}^{\prime} \bar{w}_{t}\right|=$ $\left|w_{t}\right|$, then replace $w_{t}$ by $w_{t}^{\prime}=w_{t} \overline{w_{\lambda}^{\prime}}$. Note that $\left|w_{t} \overline{w_{\lambda}^{\prime}}\right|<\left|w_{t}\right|$ contradicts the fact that $X$ is Nielsen reduced. Next consider $w_{t}^{\prime}$. If there is some $w_{\mu}^{\prime} \in X^{\prime}$ such that $\left|w_{\mu}^{\prime} \overline{w_{t}^{\prime}}\right|=\left|w_{t}^{\prime}\right|$, then replace $w_{t}^{\prime}$ by $w_{t}^{\prime \prime}=w_{t}^{\prime} \overline{w_{\mu}^{\prime}}$. Since the right half of $w_{\mu}^{\prime}$ is isolated in $X^{\prime}, \lambda<\mu$. Thus it is best to choose $\lambda=\operatorname{maximum}\left\{1,2, \ldots, t-1 /\left|w_{\lambda}^{\prime} \bar{w}_{t}\right|=\left|w_{t}\right|\right\}$. Continuing in this way we arrive at a $w_{t_{1}}$ from which the right half of every element in $X^{\prime}$ is isolated. However, $\bar{w}_{t_{1}}$ might end with the right half of some element in $X^{\prime}$. If so, then repeat the above procedure to obtain $\bar{w}_{t_{2}}$. Now the right halves of elements from $X^{\prime}$ are isolated from $w_{t_{2}}$ and $\bar{w}_{t_{2}}$, that is $\left|w_{\lambda}^{0} \bar{w}_{t_{2}}\right|>\left|w_{t_{2}}\right|$ and $\left|w_{\lambda}^{\prime} w_{t_{2}}\right|>\left|w_{t_{2}}\right|$ for all $w_{\lambda}^{\prime} \in X^{\prime}$.

Finally we need to assure that at least one side of $w_{t_{2}}$ is isolated in $X^{\prime}$. If $w_{t_{2}}$ 's left or right half is isolated in $X^{\prime}$, then $X^{\prime} \cup\left\{w_{t_{2}}\right\}$ is fully Nielsen reduced. Assume that neither side of $w_{t_{2}}$ is isolated. Then there must exist $w_{\lambda_{1}}^{\prime}$ and $w_{\lambda_{2}}^{\prime}$ in $X^{\prime}$ such that $\left|w_{t_{2}} w_{\lambda_{1}}^{\prime}\right|=\left|w_{\lambda_{1}}^{\prime}\right|=\left|w_{t_{2}}\right|$ and $\left|\bar{w}_{t_{2}} w_{\lambda_{2}}^{\prime}\right|=\left|w_{\lambda_{2}}^{\prime}\right|=\left|w_{t_{2}}\right|$. We will modify $X^{\prime}$ so as to isolate the right half of $w_{t_{2}}$ from those $\bar{w}_{\lambda}^{\prime}$ that end with the right half of $w_{t_{2}}$. If $\left|w_{\lambda}^{\prime}\right|=\left|w_{t_{2}} w_{\lambda}^{\prime}\right|$, then let $w_{\lambda}^{\prime \prime}=w_{t_{2}} w_{\lambda}^{\prime}$; otherwise let $w_{\lambda}^{\prime \prime}=w_{\lambda}^{\prime}$. Then $\left\{w_{1}^{\prime \prime}, w_{2}^{\prime \prime}, \ldots, w_{t-1}^{\prime \prime}, w_{t_{2}}\right\}$ is fully Nielsen reduced.

Repeat the entire procedure for $w_{t+1}, w_{t+2}, \ldots$, and $w_{n}$. Finally we obtain a set $X^{*}$ which is fully Nielsen reduced.

Theorem 4.4. If a set $X=\left\{x_{1}, x_{2}, \ldots, x_{n}\right\}$ of elements from a group $G$ with a real length function is fully Nielsen reduced, then $H=\left\langle x_{1}, x_{2}, \ldots, x_{n}\right\rangle$ is a free group.

Proof. Consider $w=w_{1} w_{2} w_{3} \cdots w_{k}$ where each $w_{i} \in\{x, \bar{x}\}$ for some $x \in$ $X, w_{i} \neq 1$, and $w_{i+1} \neq \bar{w}_{i}$ for $1 \leq i \leq k-1$. It suffices to show that $w \neq 1$ so we will check that the hypotheses of Theorem 4.2 hold. Let $1 \leq i<n$. Since $X$ is Nielsen reduced, $c\left(w_{i}, \overline{w_{i+1}}\right) \leq \operatorname{minimum}\left\{1 / 2\left|w_{i}\right|, 1 / 2\left|w_{i+1}\right|\right\}$, provided $w_{i+1} \neq$ $w_{i}$. And if $w_{i+1}=w_{i}$, then $A_{0}$ implies $c\left(w_{i}, \bar{w}_{i}\right)<1 / 2\left|w_{i}\right|$. Thus (4.2a) is satisfied. 
We now show that it is not possible for $c\left(w_{i-1}, \bar{w}_{i}\right)=c\left(w_{i}, \overline{w_{i+1}}\right)=1 / 2\left|w_{i}\right|$. If $w_{i-1}=w_{i}$ then $c\left(w_{i-1}, \widehat{w_{i}}\right)<1 / 2\left|w_{i}\right|$ by $A_{0}$. Similarly, $w_{i+1}=w_{i}$ implies $c\left(w_{i}, \overline{w_{i+1}}\right)<1 / 2\left|w_{i}\right|$. Assume $w_{i-1} \neq w_{i}$ and $w_{i+1} \neq w_{i}$. Since $X$ is fully Nielsen reduced, the left or right half of $w_{i}$ must be isolated in $X$. If $w_{i}$ 's left half is isolated, then $c\left(w_{i-1}, \bar{w}_{i}\right)<1 / 2\left|w_{i}\right|$ and if its right half is isolated, then $c\left(w_{i}, \overline{w_{i+1}}\right)<1 / 2\left|w_{i}\right|$. Thus $c\left(w_{i-1}, \bar{w}_{i}\right)=c\left(w_{i}, \overline{w_{i+1}}\right)=1 / 2\left|w_{i}\right|$ holds for no $i$. It follows that the third hypothesis is valid vacuously. By invoking Theorem 4.2 we can conclude that $w \neq 1$. Hence $H$ is a free group with free generators $X-\{1\}$.

Next we want to define a reduction sequence for a finite subset of elements from $G$. Let $X=\left(x_{1}, x_{2}, \ldots, x_{n}\right)$ be a sequence of elements from $G$ such that $\left|x_{1}\right| \leq\left|x_{2}\right| \leq \cdots \leq\left|x_{n}\right|$. We define the length of $X$, denoted $|X|$, to be $\sum_{i=1}^{n}\left|x_{i}\right|$. A reduction of $X$ is an elementary Nielsen transformation applied to $X$ to obtain a new sequence $X^{\prime \prime}$ such that the length of $X^{\prime \prime}$ is shorter than that of $X$, followed by an elementary Nielsen transformation which permutes the sequence $X^{\prime \prime}$ to $X^{\prime}=$ $\left(x_{1}^{\prime}, x_{2}^{\prime}, \ldots, x_{n}^{\prime}\right)$ so that $\left|x_{1}^{\prime}\right| \leq\left|x_{2}^{\prime}\right| \leq \ldots \leq\left|x_{n}^{\prime}\right|$. Then $X^{[0]}=\left(x_{1}, \ldots, x_{n}\right)$, $X^{[1]}=\left(x_{1}^{[1]}, x_{2}^{[1]}, \ldots, x_{n}^{[1]}\right), \ldots$ where $X^{[i+1]}$ is a reduction of $X^{[i]}$ is called a reduction sequence. For some sequences of elements from $G$ this reduction process stops, that is we reach a term $X^{[N]}$ whose length cannot be reduced any further by applying elementary Nielsen transformations. For other sequences the process continues indefinitely. For example let $R$ be the additive group of real numbers with absolute value as a length function. The reduction process for $\{3, \pi\}$ is infinite.

As a corollary to the previous theorem we get

Corollary 4.5. If the reduction sequence of $X=\left(x_{1}, x_{2}, \ldots, x_{n}\right)$, a set of elements from a group $G$ with a real length function, is finite, then $H=\left\langle x_{1}, x_{2}\right.$, $\left.\cdots, x_{n}\right\rangle$ is a free group.

We want to define limit numbers for a reduction sequence. Let $X=\left(x_{1}, x_{2}\right.$, $\left.\ldots, x_{n}\right)$ be a sequence of elements from $G$ such that $\left|x_{1}\right| \leq\left|x_{2}\right| \leq \cdots \leq\left|x_{n}\right|$, and let $X^{[0]}=X, X^{[1]}, X^{[2]}, \ldots$ be a reduction sequence for $X$. For each $i, 1$ $\leq i \leq n$, consider $\left|x_{i}^{[1]}\right|,\left|x_{i}^{[2]}\right|,\left|x_{i}^{[3]}\right|, \ldots$. By our construction of a reduction sequence $\left|x_{i}^{[s]}\right| \geq\left|x_{i}^{[s+1]}\right|$ for all $s$. Thus for each $i,\left|x_{i}^{[1]}\right|,\left|x_{i}^{[2]}\right|, \ldots$ is a weakly decreasing sequence of real numbers bounded below by zero and hence has a limit. If the reduction sequence stops at the $N$ th step, define the $i$ th limit number to be $\left|x_{i}^{[N]}\right|$. And if the reduction sequence is infinite, define the ith limit number to be $\operatorname{limit}_{s \rightarrow \infty}\left|x_{i}^{[s]}\right|$.

A reduction sequence for a set of elements from $G$ is by no means unique. However, we claim that the limit numbers of a finite reduction sequence for a sequence $X$ of 
elements from $G$ depend only on $X$ itself and not on the reduction process. This proof is adapted from Magnus, Karrass, and Solitar [2, Corollary 3.1, p. 128].

Proposition 4.6. Suppose $S$ and $T$ are two reduction sequences for $X=\left(x_{1}\right.$, $\left.x_{2}, \ldots, x_{n}\right)$, a subset of elements from a group $G$ with a real length function. If $S$ and $T$ stop after a finite number of steps, their limit numbers are equal.

Proof. Let $H$ denote the subgroup of $G$ generated by $X$. And let $\left(y_{1}^{\prime}, y_{2}^{\prime}, \ldots, y_{n}^{\prime}\right)$ and $\left(z_{1}^{\prime}, z_{2}^{\prime}, \ldots, z_{n}^{\prime}\right)$ be the last points in the reduction sequences $S$ and $T$ respectively. By Theorem 4.3 we can obtain sets $Y=\left\{y_{1}, y_{2}, \cdots, y_{n}\right\}$ and $Z=\left\{z_{1}, z_{2}, \cdots, z_{n}\right\}$ which are fully Nielsen reduced and generate $H$. Let $H_{k}=\langle x \in H|| x \mid \leq k\rangle, Y_{k}=\langle y \in Y|| y \mid \leq k\rangle$, and $Z_{k}=\langle z \in Z|| z \mid \leq k\rangle$. We claim that $H_{k}=Y_{k}=Z_{k}$. Clearly $Y_{k} C$ $H_{k}$. We assume that $H_{k} \not \subset Y_{k}$ and derive a contradiction. Since $H_{k} \not \subset Y_{k}$, there exists some $x$ in $H_{k}$ such that $|x| \leq k$ and $x \notin Y_{k}$. Since $x \in$ $\left\langle y_{1}, y_{2}, \ldots, y_{n}\right\rangle=H$, we can write $x=w_{1} w_{2} \cdots w_{m}$, where $w_{i} \in\left\{y_{j}, \bar{y}_{j} \mid 1 \leq\right.$ $j \leq n\}, w_{i} \neq 1$, and $w_{i+1} \neq \bar{w}_{i}$ for $1 \leq i<m$. In view of the proof of Theorem 4.4 we know that $w_{1}, w_{2}, \ldots, w_{m}$ satisfy the hypotheses of Theorem 4.2. Therefore $|x|=\sum_{i=1}^{m}\left|w_{i}\right|-2 \sum_{i=1}^{m-1} c\left(w_{i}, \frac{m}{w_{i+1}}\right)$. For $1 \leq j \leq m$,

$$
|x|=\left|w_{j}\right|+\sum_{i=1}^{j-1}\left[\left|w_{i}\right|-2 c\left(w_{i}, \overline{w_{i+1}}\right)\right]+\sum_{i=j+1}^{m}\left[\left|w_{i}\right|-2 c\left(w_{i-1}, \bar{w}_{i}\right)\right] .
$$

Since $c\left(w_{i}, \overline{w_{i+1}}\right) \leq$ minimum $\left\{1 / 2\left|w_{i}\right|, 1 / 2\left|w_{i+1}\right|\right\}$, the above expression yields $|x| \geq$ $\left|w_{j}\right|$. Now at least one $b, 1 \leq b \leq m$, is such that $\left|w_{b}\right|>k$; for if not, $x \in Y_{k}$. But then $|x| \geq\left|w_{b}\right|>k$, contradicting our choice of $x$. Thus $Y_{k}=H_{k}$. Similarly $Z_{k}=H_{k}$. By Corollary 4.5, $Y-\{1\}$ and $Z-\{1\}$ freely generate the free group $H_{k}$. Since the rank of $H_{k}$ is unique, $Y$ and $Z$ have the same number of nontrivial elements $w$ ith length $\leq k$ and hence of length $k$.

We want to discuss briefly the limit numbers of infinite reduction sequences. It is easy to see that without further qualifications two infinite reduction sequences for a finite set $X$ of elements from $G$ need not have the same limit numbers. Suppose $G=A_{1} * A_{2} * A_{3}$ where $A_{i}$ is the additive group of real numbers with absolute value as a length function, and give $G$ the length function of Proposition 1.2. Take $X=\{(2,1),(\pi, 1),(e, 2),(3,2)\}$ where $(r, k)$ is the real number $r$ from $A_{k}$. We can formulate an infinite reduction sequence for $X$ which reduces $(2,1)$ and $(\pi, 1)$ but leaves $(e, 2)$ and $(3.2)$ alone; its limit numbers would be $0,0, e, 3$. We can also formulate an infinite reduction sequence which reduces both $\{(2,1),(\pi, 1)\}$ and $\{(e, 2),(3,2)\}$ and has limit numbers $0,0,0,0$. Thus in contrast to a finite reduction sequence, the limit numbers of an infinite sequence do not depend on $X$ alone. In this paper we are mainly concerned with 
reduction sequences of pairs of elements, so this situation does not present any difficulty. We do feel that a more restrictive type of reduction sequence can be defined in such a way that its limit numbers would depend only on $X$. But the problems involved are major, so we did not pursue this question any further.

We close this section with a result about reduction sequences for a pair of elements.

Proposition 4.7. Let $S$ be a reduction sequence for a pair of elements from a group $G$ with a real length function. If all pairs beyond some fixed pair have equal first coordinates, then the reduction sequence is finite.

Proof. Let $\alpha$ and $\beta$ where $\alpha \leq \beta$ be the limit numbers of $S$. Assume that all pairs beyond $(a, y)$ have their first coordinate equal to $a$, that is the sequence takes the form $(a, y),\left(a, y_{1}\right),\left(a, y_{2}\right), \ldots$ with $\left|y_{i+1}\right|<\left|y_{i}\right|$ but $\left|y_{i}\right| \geq$ $|a|$ for all $i$. Then $\alpha=|a|$ and there exists an integer $N$ such that, for $n \geq N$, $\left|y_{n}\right|<\beta+\alpha-2 d$ where $d=c(a, \bar{a})$. Let $z=y_{N}$ and $z_{i}=y_{N+i}$ for $i \geq 1$. Then the sequence from $\left(a, y_{N}\right)$ is as follows:

$$
(a, z),\left(a, z_{1}\right),\left(a, z_{2}\right), \ldots \text {. }
$$

We will show that it stops in a finite number of steps. Without loss of generality assume $z_{1}=a z$ and $|a z| \leq \operatorname{minimum}\{|\bar{a} z|,|\bar{a} z|, a \bar{z} \mid\}$. Then $z^{2}$ is $a a z, a z a$, or $a z \bar{a}$. If $z_{2}=a a z$, then $c(a, \bar{a})<c(a, \bar{a} z)$, so $\mathrm{A}_{4}$ implies $c(a, \bar{a})=c(\bar{a}, \bar{a} z)$. From this it follows that $|a z|=|z|-|a|+2 d<\beta$ since $|z|<\beta+\alpha-2 d$. But this is a contradiction. Therefore $z_{2}$ is either $a z a$ or $a z \bar{a}$. Since the arguments are similar for both of these, we will only present the details for $z_{2}=a z a$. In this case $z_{3}$ is $a a z a, z a$, or azaa. Since $\left|z_{3}\right|<|a z| \leq|z a|, z_{3} \neq z a$. And if $z_{3}=a z a a, c(a, \bar{a})<c(a z a, \bar{a})$, so $A_{4}$ implies $c(a, \bar{a})=c(a z a, a)$. But then $|a z a|=-|a|+|a z|+2 d<\beta$, a contradiction. Finally suppose $z_{3}=a a z a$. We need first to show that $|z a|<|z|$. It is easy to see that $c(a z, \bar{a})=c(z, \bar{a})$. For if $c(z, \bar{a})<c(a z, \bar{a})$, then $\mathrm{A}_{4}$ implies $c(z, \bar{a})=c(a z, z)$ and $|z a|+|a z|=$ $2|a|$. This is impossible; $z_{2}=a z$ was chosen so that $|a z| \leq|z a|$, and $\left|z_{2}\right|>$ $|a|$ or the sequence stops. And if $c(a z, \bar{a})<c(z, \bar{a})$, then $\mathrm{A}_{4}$ implies $c(a z, \bar{a})$ $=c(a z, z)$ so $|a z a|+|z|=2|a|$. This is a contradiction; $\left|z_{3}\right|=|a z a|>|a|$ or the sequence stops and $\left|z_{3}\right|<\left|z_{2}\right|<|z|$. Since $|a z a|<|a z|, c(a z, \bar{a})>1 / 2|a|$. Hence $c(z, \bar{a})>1 / 2|a|$ or equivalently $|z a|<|z|$. Since $|a a z a|<|a z a|, c(a, \bar{a})$ $<c(a, \bar{a} \bar{z} \bar{a}) ; A_{4}$ implies $c(a, \bar{a})=c(\bar{a}, \bar{a} \bar{z} \bar{a})$ so $|a z a|=|z a|-|a|+2 d$. Since $|z a|<|z|$ and $|z|<\beta+|a|-2 d$, it follows that $|a z a|<\beta$. But this is a contradiction. Therefore the pair $\left(a, z_{2}\right)$ must be Nielsen reduced so the reduction process stops.

5. Two-generator groups with real length functions. In this section we 
determine the structure of a group which is generated by two elements and has a real length function. Throughout this section let $G$ be a group equipped with a real length function $\mid 1$. In view of Corollary 4.5 if the reduction process for $\{x, y\}$ where $x, y \in G$ is finite, then $\langle x, y\rangle$ is a free group. In this section we prove that if the reduction process for $\{x, y\}$ continues indefinitely then $\langle x, y\rangle$ is abelian.

Proposition 5.1. If a reduction sequence for $x, y \in G$ is infinite with limit numbers $\alpha$ and $\beta$, then $\alpha=\beta$.

Proof. Let $x, y \in G$ with $|x| \leq|y|$. Let $(x, y),\left(x_{2}, y_{2}\right), \ldots$ be a reduction sequence for $(x, y)$ with limit numbers $\alpha$ and $\beta$ where $\alpha<\beta$. Since $\operatorname{limit}_{n \rightarrow \infty}\left|x_{n}\right|=\alpha<\beta$, there exists a positive integer $N$ such that, for $n \geq N,\left|x_{n}\right|$ $<\beta$. We claim that $x_{N}=x_{N+k}$ for all $k \geq 0$. The new element $p$ in the $(N+1)$ th pair has length less than that of $y_{N}$. Since $\left|x_{N}\right|<\beta,|p| \geq \beta$. Hence $y_{N+1}=p$ and $x_{N+1}=x_{N}$. Similarly $x_{N}=x_{N+k}$ for $k>1$. By Proposition 4.7 the reduction sequence is finite.

Proposition 5.2. Let $\left(x_{i}, y_{i}\right)$ be an infinite reduction sequence for $x, y \in G$ where limit ${ }_{i \rightarrow \infty}\left|x_{i}\right|=$ limit $_{i \rightarrow \infty}\left|y_{i}\right|=0$. Then $\langle x, y\rangle$ is abelian.

Proof. For an arbitrary pair of elements $g, b \in G$ let $\Gamma(g, b)=\{[g, b],[\bar{g}, b]$, $[g, \bar{b}],[\bar{g}, \bar{b}]\}$ where $[g, b]=\overline{g h} g h$, the commutator of $g$ and $h$. When we apply a Nielsen transformation to $(g, b)$ to obtain $\left(g^{\prime}, b^{\prime}\right)$, there is a one-to-one correspondence between the sets $\Gamma(g, b)$ and $\Gamma\left(g^{\prime}, b^{\prime}\right)$ such that corresponding elements are conjugates. This is straightforward to verify and known, so we omit the details. For each $g \in G$ define $f(g)=\left|g^{2}\right|-|g|$. And for each pair in our reduction sequence $\left(x_{n}, y_{n}\right)$ define $K_{n}=\operatorname{minimum}\left\{f(a) \mid a \in \Gamma\left(x_{n}, y_{n}\right)\right\}$. Since $f$ is a class function by Theorem 2.8, the above implies that $K_{i}=K_{1}$ for all $i$. Since limit ${ }_{n \rightarrow \infty}\left|x_{n}\right|=0=$ limit $_{n \rightarrow \infty}\left|y_{n}\right|$, given any $\epsilon>0$ there exists an integer $N$ such that, for all $n \geq N,\left|y_{n}\right|<1 / 4 \epsilon$. Now

$$
K_{n} \leq f\left(\bar{x}_{n} \bar{y}_{n} x_{n} y_{n}\right) \leq\left|\bar{x}_{n} \bar{y}_{n} x_{n} y_{n}\right| \leq 2\left|x_{n}\right|+2\left|y_{n}\right| \leq \epsilon .
$$

Hence $K_{1}=0$ so $x$ and $y$ commute.

We turn now to the case when every reduction sequence for $(x, y)$ is infinite with limit numbers $\alpha=\beta>0$. Let $\left(x_{i}, y_{i}\right)$ be a reduction sequence for $(x, y)$. There are several parts to this case. First we show that from some point on in our sequence no element occuring is cyclically reduced. Secondly we verify some simple lemmas concerning the amounts of cyclic reduction and the lengths of elements in our sequence. Thirdly we show that limit $_{i \rightarrow \infty} c\left(y_{i}, \bar{y}_{i}\right)=$ limit $_{i \rightarrow \infty} c\left(x_{i}, \bar{x}_{i}\right)=1 / 2 \alpha$. Finally we prove that $x$ and $y$ commute. 
If $a$ and $b$ are the elements of some pair in a reduction sequence for $x$ and $y$ but it is not determined whether $a$ or $b$ has the shorter length, we will use the notation $(a / b)$ for the pair.

Lemma 5.3. Let $\left(x_{i}, y_{i}\right)$ be an infinite reduction sequence for $x, y \in G$ where limit $_{i \rightarrow \infty}\left|x_{i}\right|=\operatorname{limit}_{i \rightarrow \infty}\left|y_{i}\right|=a>0$. Then given any $N$ there exists $n>N$ such that some element of $\left(x_{n}, y_{n}\right)$ is not cyclically reduced.

Proof. Let $0<\epsilon<(1 / 3) \alpha$. Choose $M$ such that $\left|y_{M}\right|<\alpha+\epsilon$. Let $x_{M}=u$ and $y_{M}=v$. Since $\{u, v\}$ is not Nielsen reduced,

$$
\text { minimum }\{|\bar{u} v|,|\bar{u} \bar{v}|,|u v|,|u \bar{v}|\}<|v| \text {. }
$$

For definiteness assume that $\bar{u} v$ is the new element in the $(M+1)$ th pair of our sequence. Again $\{u, \bar{u} v\}$ is not Nielsen reduced so the following holds:

$$
\text { minimum }\left\{\left|\bar{v} u^{2}\right|,|\bar{u} v u|,|\bar{u} v \bar{u}|\right\}<\operatorname{maximum}\{|u|,|\bar{u} v|\} \text {. }
$$

Case 1. If $\left|\bar{v} u^{2}\right|<\operatorname{maximum}\{|u|,|\bar{u} v|\}$, then $u$ is not cyclically reduced.

Suppose on the contrary that $u$ is cyclically reduced. Then $c(\bar{u}, u)<$ $c(\bar{u}, \overline{\bar{u} v})$ so $\mathrm{A}_{4}$ implies $0=c(u, \overline{\bar{u} v})$ and $|\bar{u} v|=|v|-|u|$. But $|v|-|u|<\epsilon<\alpha$ so this is a contradiction.

Case 2. If $|\bar{u} v u|<\operatorname{maximum}\{|u|,|\bar{u} v|\}$, then $v$ is not cyclically reduced.

Since $|\bar{u} v|<|v|, c(\bar{u}, \bar{v})>0$. It suffices to show that $c(v, \bar{u})>0$, for $\mathrm{A}_{4}$ then implies $c(v, \bar{v})>0$. Assume on the contrary that $c(v, \bar{u})=0$, so $|v u|=$ $|v|+|u|$. By Proposition 2.1, $|\bar{u} v u| \geq|v u|-|u|=|v|$. But this contradicts our hypothesis since $|v| \geq \operatorname{maximum}\{|u|,|\bar{u} v|\}$.

Case 3. If $|\bar{u} v \bar{u}|<\operatorname{maximum}\{|u|,|\bar{u} v|\}$, then $\bar{u} v$ is not cyclically reduced.

In view of $\mathrm{A}_{4}$ it suffices to show that $c(u, \overline{\bar{u} v})>0$ and $c(\bar{u} v, u)>0$. If $c(u, \overline{\bar{u} v})=0$, then $|\bar{u} v|=|v|-|u|<\epsilon<\alpha$, a contradiction. And $c(\bar{u} v, u)=0$ implies $|\bar{u} v \bar{u}|=|\bar{u} v|+|u|$, a contradiction of the hypothesis.

Therefore, given any $N$ there exists $n>N$ such that some element of $\left(x_{n}, y_{n}\right)$ is not cyclically reduced.

Lemma 5.4. Let $\left(x_{i}, y_{i}\right)$ be an infinite reduction sequence for $x, y \in G$ where limit ${ }_{i \rightarrow \infty}\left|x_{i}\right|=\operatorname{limit}_{i \rightarrow \infty}\left|y_{i}\right|=\alpha>0$. Furthermore assume that for all $i$, $\left(x_{i+1}, y_{i+1}\right)=\left(x_{i} / z\right)$ where $z$ bas the shortest length of all the possible choices. Then given any $N$ there exists $n>N$ such that neither element of $\left(x_{n}, y_{n}\right)$ is cyclically reduced.

Proof. Let $0<\epsilon<(1 / 3) \alpha$. Choose $Q$ such that $\left|y_{Q}\right|<\alpha+\epsilon$. Let $x_{Q}=$ $u$ and $y_{Q}=v$. Let $\bar{u} v$ be the new element in the $(Q+1)$ th pair. By our hypothesis this pair is $(u / \bar{u} v)$ where $|\bar{u} v| \leq$ minimum $\{|u v|,|u \bar{v}|,|\bar{u} \bar{v}|\}$. We 
consider three cases as in the previous lemma.

Case 1. $\bar{v} u u$ is a coordinate of the second pair following $(u, v)$.

By Lemma 5.3 we know that $u$ is not cyclically reduced. We show that neither is $v$. Suppose $c(u, v)>0$. Since $c(u, \bar{u}) \neq 0, c(\bar{u}, v)>0$ by $A_{4}$. And since $|\bar{u} v|<|v|, c(\bar{u}, \bar{v})>0$. Axiom $A_{4}$ then implies $c(v, \bar{v})>0$. Therefore if $c(u, v)>0$, we are done.

We show that $c(u, v)=0$ leads to a contradiction. Assume $c(u, v)=0$, from which it follows by $\mathrm{A}_{4}$ that $c(\bar{u}, v)=0$ also. Let $d=c(u, \bar{u})$. Note that $\alpha-\epsilon<$ $2 d<\alpha+\epsilon$. If $d<1 / 2 \alpha$ then $c(u, \bar{u})<c(\bar{v} u, \bar{u})$. Axiom $A_{4}$ implies $d=c(u, \bar{v} u)$, so $|\bar{v} u|=|v|-|u|+2 d<\epsilon+2 d$. Since $|\bar{v} u|>\alpha, \alpha-\epsilon<2 d<\alpha$. And if $d \geq 1 / 2 \alpha$, then $a \leq 2 d<|u|<\alpha+\epsilon$.

By Proposition 2.6, the lengths $\left|u^{p}\right|$ are unbounded so we can find a positive integer $p$ such that $\left|u^{p}\right|>2|v|$; and by Proposition 2.1, $c\left(\bar{v}, \bar{u}^{q}\right) \leq|v|$ for all $q$. It follows that $\left|\bar{v} u^{p}\right|>|v|$. Let $n$ be the integer such that $\left|\bar{v} u^{n+1}\right|<\left|\bar{v} u^{n}\right|<\ldots$ $<|\bar{v} u|<|v|$ and $\left|v u^{n+2}\right| \geq\left|\bar{v} u^{n+1}\right|$. Since $|\bar{v} u|<|v|$, we know that $n \geq 0$. We want to determine $\left|\bar{v}^{m}\right|$ for $1 \leq m \leq n$. If $c\left(\overline{v u}^{m}, \bar{u}\right) \leq c(u, \bar{u})=d$, then $\left|\bar{v} u^{m+1}\right|$ $\geq\left|\bar{v} u^{m}\right|+|u|-2 d$. But this contradicts the fact that $\left|\bar{v} u^{m+1}\right|<\left|\bar{v} u^{m}\right|$ since $m \leq$ $n$. Therefore $c(u, \bar{u})<c\left(\bar{v} u^{m}, \bar{u}\right)$, so $A_{4}$ implies $\left|\bar{v} u^{m}\right|=\left|\bar{v} u^{m-1}\right|-|u|+2 d=$ $|v|-m|u|+2 m d$. Note that if $\bar{v} u^{n}$ and $\bar{v} u^{n+1}$ occur in the sequence, then $\left|\bar{v} u^{n+1}\right| \neq\left|u^{n+1}\right|-|v| ;$ for $\left|\dot{u}^{n+1}\right|-|v|=(n+1)|u|-2 n d-|v|=-\left|\bar{v} u^{n}\right|+|u|<$ $\epsilon<a$. Also if $\bar{v} u^{n}$ and $\bar{v} u^{n+2}$ occur in the sequence, then $\left|\bar{v} u^{n+2}\right| \neq\left|u^{n+2}\right|-$ $|v|$; for $\left|u^{n+2}\right|-|v|=(n+2)|u|-2(n+1) d-|v|=|u|-\left|\bar{v} u^{n}\right|+|u|-2 d<2 \epsilon<a$.

Any pair $\{s, t\}$ in $\left\{\left\{u, \bar{v} u^{m}\right\},\left\{\bar{v} u^{m}, \bar{v} u^{m-1}\right\},\left\{\bar{v} u^{m}, \bar{v} u v\right\}\right\}$ where $m$ is a positive integer will be called normal if maximum $\{|s|,|t|\}<|v|$. Since $\left|\bar{v} u^{p}\right|>|v|$, there are only a finite number of distinct normal pairs. Suppose then that every pair from $(u, v)$ through $\left(x_{N}, y_{N}\right)$ is normal but the $(N+1)$ th pair is not normal. If $u \notin\left\{x_{M}, y_{M}\right\}$ where $M \leq N$, then there exists an integer $r$ between 1 and $M+1$ such that the $r+1$ terms following $(u, v)$ are $(u, \bar{v} u),\left(u, \bar{v} u^{2}\right), \ldots,\left(u, \bar{v} u^{r \cdots 1}\right)$, $\left(\bar{v} u^{r}, u\right),\left(\bar{v} u^{r} / \bar{v} u^{r+1}\right)$. And since maximum $\left\{\left|\bar{v} u^{r}\right|,\left|\bar{v} u^{r+1}\right|\right\}<|u|$, the length of any element occuring in the sequence beyond $\left(\bar{v} u^{r} / \bar{v} u^{r+1}\right)$ is less than the length of $u$. Let $M<N$. Then the $M$ th and $(M+1)$ th terms are both normal. Now

$$
\begin{aligned}
& \begin{array}{l}
\left(x_{M}, y_{M}\right)=\left(u / \bar{v} u^{m}\right) \quad \text { implies } \\
\left(x_{M+1}, y_{M+1}\right) \in\left\{\left(u / \bar{v} u^{m+1}\right),\left(u / \bar{v} u^{m-1}\right),\left(\bar{v} u^{m} / \bar{v} u^{m-1}\right),\left(\bar{v} u^{m} / \bar{v} u^{m+1}\right)\right\} ; \\
\left(x_{M}, y_{M}\right)=\left(\bar{v} u^{m} / \bar{v} u^{m-1}\right) \quad \text { implies } \\
\qquad\left(x_{M+1}, y_{M+1}\right) \in\left\{\left(\bar{v} u v / \bar{v} u^{m}\right),\left(\bar{v} u v / \bar{v} u^{m-1}\right)\right\} ; \\
\left(x_{M}, y_{M}\right)=\left(\bar{v} u^{m} / \bar{v} u v\right) \quad \text { implies } \\
\left(x_{M+1}, y_{M+1}\right) \in\left\{\left(\bar{v} u^{m-1} / \bar{v} u v\right),\left(\bar{v} u^{m+1} / \bar{v} u v\right),\left(\bar{v} u^{m-1} / \bar{v} u^{m}\right),\left(\bar{v} u^{m+1} / \bar{v} u^{m}\right)\right\} .
\end{array}
\end{aligned}
$$


From this it is easy to see if $\bar{v} u^{s}$ occurs in the Sth pair where $S \leq N$, then $\bar{v} u^{m}$ for all $m<s$ occurs in some pair prior to the Sth pair.

Suppose $\bar{v} u^{m} \in\left\{x_{N}, y_{N}\right\}$ but $\bar{v} u^{m+1} \notin\left\{x_{N}, y_{N}\right\}$. First assume that $\left(x_{N}, y_{N}\right)$ $=\left(\overline{v u}^{m}, \bar{v}^{m-1}\right)$. Then the new element $z$ in the $(N+1)$ th pair is either $\bar{v} u^{m} \overline{v u}^{m-1}$ or $\bar{v} u^{m-1} \bar{v}^{m}$. Suppose $z=\overline{v u}^{m} \bar{v}^{m-1}$. If $c\left(\bar{v}^{m}, v\right)>c(u, v)=0$, then $\mathrm{A}_{4}$ implies $\left|\bar{v}^{m}\right|=\left|\bar{v} u^{m-1}\right|-|u| \leq \epsilon$, a contradiction. Therefore $0=$ $c\left(\bar{v} u^{m}, v\right)<c\left(\bar{v} u^{m}, \overline{v u^{m-1}}\right) ; A_{4}$ implies $\left|\bar{v} u^{m-1}\right|=\left|u^{m-1}\right|-|v|$, contrary to what we established earlier provided $m-1 \leq n+2$. Suppose $z=\bar{v} u^{m-1} \bar{v} u^{m}$. If $c\left(\bar{v}^{m-1}, v\right)>c(u, v)=0$, then $A_{4}$ implies $\left|\bar{v} u^{m-1}\right|=\left|\bar{v}^{m-2}\right|-|u| \leq \epsilon$, a contradiction. Thus $0=c\left(\bar{v} u^{m-1}, v\right)<c\left(\bar{v} u^{m-1}, \overline{\bar{v} u^{m}}\right) ; A_{4}$ implies $\left|\bar{v} u^{m}\right|=\left|u^{m}\right|-|v|$, contrary to what we established earlier provided $m \leq n+2$. Now assume that $m$ $>n+2$. Then $\bar{v} u^{n+3}$ occurs in some term of the normal part of the sequence. Let $R$ be the term in which $\bar{v} u^{n+3}$ first appears. Then the $R$ th term is necessarily $\left(u / \bar{v} u^{n+3}\right),\left(\bar{v} u^{n+2} / v u^{n+3}\right)$, or $\left(\bar{v} u^{n+3}, \bar{v} u v\right)$. Now $\bar{v} u^{n+1}$ must occur in the Sth term where $S<R$. Since $\bar{v} u^{n+1}$ does not occur in the $R$ th term, the fact that we always replace the element of the larger length implies that $\left|\bar{v} u^{n+3}\right|<\left|\bar{v} u^{n+1}\right|$. We claim that this impossible. Let $T=c\left(\bar{v} u^{n+1}, \bar{u}\right)+c(u, \bar{u})$. If $T<|u|$, then Proposition 2.4 implies $c\left(\bar{v} u^{n+2}, \bar{u}\right)=c(u, \bar{u})$. Thus

$$
\left|\bar{v} u^{n+3}\right|=\left|\bar{v} u^{n+2}\right|+|u|-2 d>\left|\bar{v} u^{n+2}\right| \geq\left|\bar{v} u^{n+1}\right| \text {. }
$$

If $T \geq|u|$, then $c\left(\bar{v} u^{n+1}, \bar{u}\right) \geq|u|-d$ so $\left|\bar{v} u^{n+2}\right| \leq\left|\bar{v} u^{n+1}\right|-|u|+2 d<\left|\bar{v} u^{n+1}\right|$, contrary to our choice of $n$.

Secondly assume that $\left(x_{N}, y_{N}\right)=\left(\overline{v u}^{m} / \bar{v} u v\right)$. Then the new element $z$ in the $(N+1)$ th pair is either $\bar{v} \bar{u} \bar{u}^{m} v$ or $\bar{v} u v \bar{u}^{m} v$. Suppose $z=\bar{v} \bar{u} v \bar{u} m v$ so $c\left(\bar{v} \bar{u} v, \overline{\bar{u}}^{m} v\right)>0$. Since $c\left(v, \bar{v} u^{m}\right)=0$ as show $\mathrm{n}$ above, $\mathrm{A}_{4}$ implies $|\bar{v} \bar{u}|=|\bar{v} \bar{u}|-$ $|v|$. Since $|\bar{v} u| \leq|\overline{v u}|, c(\bar{v}, u) \leq c(\bar{v}, \bar{u})$. If equality holds, then $|\bar{v} \bar{u} v|=|\bar{v} u|-$ $|v|<0$, a contradiction; if the inequality is strict, then $A_{4}$ implies $|\bar{v} u|=|u|+$ $|v|-2 d$, in which case $|\bar{v} u v|=|u|-2 d<\epsilon$, also a contradiction. Suppose $z=$ $\bar{v} u v \bar{u}^{m} v$. Then $0=c\left(v, \bar{v}^{m}\right)<c\left(\bar{v} u v, \overline{\bar{u}}^{m} v\right)$, so $A_{4}$ implies $|\bar{v} u v|=|\bar{v} u|-|v|<$ 0 , a contradiction.

Thirdly assume $\left(x_{N}, y_{N}\right)=\left(u / \bar{v} u^{m}\right)$. The new element $z$ of the $(N+1)$ th pair is either $u \bar{v} u^{m}$ or $\bar{u} \overline{v u}^{m}$. If the former $0=c(u, v)<c\left(u, \overline{\bar{v} u^{m}}\right)$, and if the latter $0=c(\bar{u}, v)<c\left(\bar{u}, \overline{\bar{v} u^{m}}\right)$. By $\mathrm{A}_{4}, 0=c\left(v, \overline{\bar{v} u^{m}}\right)$ in both cases so $\left|\bar{v} u^{m}\right|=$ $\left|u^{m}\right|-|v|$. If $m \leq n+2$, then this contradicts what we established earlier. Assume now that $m>n+2$. Let $R$ be the term in which $\bar{v} u^{n+1}$ first occurs. Since $R<N$ and $u \in\left\{x_{N}, y_{N}\right\}$, the Rth term is necessarily $\left(u, \bar{v} u^{n+1}\right)$ and the $(R+1)$ th is $\left(u / \bar{v} u^{n+2}\right)$. But $\left|\bar{v} u^{n+2}\right| \geq\left|\bar{v} u^{n+1}\right|$ by our choice of $n$. Thus we have our desired contradiction. Hence $c(u, v)>0$ and $v$ is not cyclically reduced. 
Case 2. $\bar{u} v u$ is a coordinate of the second pair after $(u, v)$.

By Lemma 5.3, $v$ is not cyclically reduced. We claim that neither is $u$. We assume the contrary and derive a contradiction. Since $c(\bar{u}, \bar{v})>0$ as $|\bar{u} v|<|v|$, $\mathrm{A}_{4}$ implies $0=c(u, v)$. And since $c(v, v)>0, \mathrm{~A}_{4}$ implies $0=c(u, v)$.

Let $d=c(v, v)$. We claim that $d \geq 1 / 2 a$. Assume on the contrary that $d<$ $1 / 2 \alpha$. Since $|\bar{u} v|\left\langle|v|, c(\bar{u}, v)>1 / 2|u|>1 / 2 \alpha>d=c(v, \bar{v})\right.$. Axiom $A_{4}$ implies $d=$ $c(\bar{u}, v)$. Since $|\bar{u} v u|<\operatorname{maximum}\{|u|,|\bar{u} v|\}, c(\bar{u} v, \bar{u})>1 / 2 \alpha>c(\bar{u}, v)$. Axiom $\mathrm{A}_{4}$ implies $c(\bar{u} v, v)=d$. But it follows that $|\bar{u} v|=|u|-|v|+2 d \leq 2 d<\alpha$, a contradiction. Therefore $1 / 2 \alpha \leq d$.

Let $n$ be such that $\left|\bar{v}^{n+1} u\right|<\left|\bar{v}^{n} u\right|<\ldots<|\bar{v} u|<|v|$ and $\left|\bar{v}^{n+2} u\right| \geq$ $\left|\bar{v}^{n+1} u\right|$. We want to determine $\left|\bar{v}^{m} u\right|$ for $1 \leq m \leq n$. If $c\left(\bar{v}, \overline{\bar{v}^{m} u}\right) \leq c(\bar{v}, \bar{v})$, then $\left|\bar{v}^{m+1} u\right| \geq|\bar{v}+| \bar{v}^{m} u|-2 d>| \bar{v}^{m} u \mid$, a contradiction. Therefore $c(\bar{v}, v)<$ $c\left(\bar{v}, \overline{\bar{v}^{m} u}\right)$, from which it follows by $\mathrm{A}_{4}$ that $d=c\left(v, \overline{\bar{v}^{m} u}\right)$. Therefore $\left|\bar{v}^{m} u\right|=$ $\left|\bar{v}^{m-1} u\right|-|v|+2 d=|u|-m|v|+2 m d$. Also note that if $\bar{v}^{n+1} u$ and $\bar{v}^{n} u$ occur in the reduction sequence, then $\left|\bar{v}^{n+1} u\right| \neq\left|v^{n+1}\right|-|u|$; for $\left|\bar{v}^{n+1} u\right|>\alpha$ and $\left|v^{n+1}\right|-|u|=|v|-\left|\bar{v}^{n} u\right|<\epsilon<\alpha$. Similarly if $\bar{v}^{n+2} u$ and $\bar{v}^{n} u$ occur in the sequence, then $\left|\bar{v}^{n+2} u\right| \neq\left|\bar{v}^{n+2}\right|-|u|$; for $\left|\bar{v}^{n+2} u\right|>a$ but $\left|\bar{v}^{n+2}\right|-|u|=|v|-$ $2 d+|v|-\left|\bar{v}^{n} u\right|<2 \epsilon<\alpha$.

The second pair after $(u, v)$ is either $(u / \bar{u} v u)$ or $(\bar{v} u / \bar{u} v u)$. Suppose it is the former. Then the new element $z$ of the third pair after $(u, v)$ is $v u, \bar{u}^{2} v u$, or $\bar{u}^{2} \bar{v} u$. Since $|v u| \geq|\bar{u} v|, z$ is not equal to $v u$. Let $w=\bar{u} v u$ if $z=\bar{u}^{2} v u$, and let $w=\bar{u} \bar{v} u$ if $z=\bar{u}^{2} \bar{v} u$, then $0=c(u, \bar{u})<c(\bar{u}, \bar{w})$. Axiom $A_{4}$ and $|\bar{u} v| \leq|v u|$ imply that $|w| \leq|v u|-|u|$. Since $c(v, \bar{u}) \leq c(\bar{u}, \bar{v}),|v u| \leq|v|+|u|-2 d$. It follows that $|w| \leq \epsilon$, a contradiction. Therefore the second pair after $(u, v)$ is $(\bar{v} u / \bar{u} v u)$. Now the lengths $\left|\bar{v}^{p} u\right|$ are unbounded. Let $r$ be the integer such that the sequence from $(u, v)$ on is as follows:

$$
(u, v),(\bar{v} u, u),(\bar{u} v u, \bar{v} u),\left(\bar{u} v u, \bar{v}^{2} u\right),\left(\bar{u} v u, \bar{v}^{3} u\right), \ldots,\left(\bar{u} v u, \bar{v}^{r-1} u\right),\left(\bar{u} v u / \bar{v}^{r} u\right)
$$

and the next pair, say the Rth pair, is not $\left(\bar{u} v u / \bar{v}^{r+1} u\right)$. The new element $z$ in the Rth pair is $\bar{u} v u \bar{v}^{r} u, \bar{u} \bar{v}^{r} u \bar{v}^{r} u$, or $\bar{v}^{r+1} u$. First note that $c(\bar{u} v u, v)=0=$ $c(\bar{u} \bar{v} u, v)$. Since $c(u, v)=0, c(\bar{u} v u, v)>0$ implies by $A_{4}$ that $|\bar{u} v u|=|\bar{u} v|-$ $|u| \leq 0$, a contradiction. And $c(\bar{u} \bar{u}, v)>0$ implies by $A_{4}$ that $|\overline{u v u}|=|\overline{u v}|-|u|$; but we showed above that $|v u|-|u|<\epsilon$ so this is also a contradiction. If $z=$ $\bar{u} v u \bar{v}^{r} u$, then $0=c(\bar{u} v u, v)<c\left(\bar{u} v u, \overline{\bar{v}^{r} u}\right)$; and if $z=\overline{u v} u \bar{v}^{r} u$ then $c(\bar{u} \bar{v} u, v)<c\left(\bar{u} \bar{v} u, \bar{v}^{r} u\right)$. In both cases $A_{4}$ implies that $\left|\bar{v}^{r} u\right|=\left|\bar{v}^{r-1} u\right|-|v| \leq 0$, a contradiction. Therefore $z=\bar{v}^{r+1} u$, and hence the $R$ th term is $\left(\bar{v}^{r} u{ }_{v}^{r+1} u\right)$. The new term in the $(R+1)$ th pair is either $\bar{v}^{r} u \bar{v}^{r+1} u$ or $\bar{v}^{r+1} u \bar{v}^{r} u$. First note that $c\left(\bar{v}^{r} u, v\right)=0=c\left(\bar{v}^{r+1} u, v\right)$. Since $c(u, v)=0, c\left(\bar{v}^{r} u, v\right)>0$ implies by $A_{4}$ that $\left|\bar{v}^{r} u\right|=\left|v^{r}\right|-|u|$ and $c\left(\bar{v}^{r+1} u, v\right)>0$ implies by $A_{4}$ that $\left|\bar{v}^{r+1} u\right|=$ 
$\left|v^{r+1}\right|-|u|$. Since $\left|\bar{v}^{r} u\right|<\left|\bar{v}^{r-1} u\right|<\cdots<\left|\bar{v}^{2} u\right|<|\bar{v} u|$ by our definition of $r, r \leq$ $n+1$. Thus by what we established earlier $\left|\bar{v}^{r} u\right| \neq\left|\bar{v}^{r}\right|-|u|$ and $\left|\bar{v}^{r+1} u\right| \neq\left|\bar{v}^{r+1}\right|-$ $|u|$. Hence $c\left(\bar{v}^{r} u, v\right)=0=c\left(\bar{v}^{r+1} u, v\right)$. Now if $z=\bar{v}^{r} u \bar{v}^{r+1} u$, then $0=c\left(\bar{v}^{r} u, v\right)<$ $c\left(\bar{v}^{r} u, \overline{\bar{v}^{r+1} u}\right)$ implying by $\mathrm{A}_{4}$ that $\left|\bar{v}^{r+1} u\right|=\left|\bar{v}^{r} u\right|-|v| \leq 0$, a contradiction. And if $z=\bar{v}^{r+1} u \bar{v}^{r} u$, then $0=c\left(\bar{v}^{r+1} u, v\right)<c\left(\bar{v}^{r+1} u, \overline{\bar{v}^{r} u}\right)$, implying by $\mathrm{A}_{4}$ that $\left|\bar{v}^{r} u\right|=$ $\left|\bar{v}^{r-1} u\right|-|v| \leq 0$, a contradiction. Therefore $c(u, \bar{u}) \neq 0$.

Case 3. $\bar{u} v \bar{u}$ is a coordinate of the second pair following $(u, v)$.

By Lemma 5.3, $\bar{u} v$ is not cyclically reduced; let $d=c(\bar{u} v, \overline{\bar{u}} v)$. We claim that $u$ is not cyclically reduced either. Since the arguments are similar to those in Case 2, we will present an outline of the development and any difficult parts but omit most details. Assume on the contrary that $c(u, \bar{u})=0$. Then $c(\bar{u}, \bar{v})>0$, $c(u, \bar{v})=0, c(u, v)=0$, and $c(\bar{u}, v)=0$. And also $a-\epsilon<2 d<a+\epsilon$.

Let $n$ be such that $\left|(\bar{u} v)^{n+1} \bar{u}\right|<\left|(\bar{u} v)^{n} \bar{u}\right|<\left|(\bar{u} v)^{n-1} \bar{u}\right|<\cdots<|\bar{u} v \bar{u}|<|v|$ and $\left|(\bar{u} v)^{n+2} \bar{u}\right| \geq\left|(\bar{u} v)^{n+1} \bar{u}\right|$. For $0 \leq m \leq n,\left|(\bar{u} v)^{m} \bar{u}\right|=|u|-m|\bar{u} v|+2 m d$. If $(\bar{u} v)^{n+1} \bar{u}$ and $(\bar{u} v)^{n} \bar{u}$ occur in the reduction sequence, then $\left|(\bar{u} v)^{n+1} \bar{u}\right| \neq\left|(\bar{u} v)^{n+1}\right|-|u|$; if $(\bar{u} v)^{n+2} \bar{u}$ and $(\bar{u} v)^{n} \bar{u}$ occur in the reduction sequence then $\left|(\bar{u} v)^{n+2} \bar{u}\right| \neq\left|(\bar{u} v)^{n+2}\right|$ $-|u|$.

The second pair after $(u, v)$ is either $(\bar{u} v \bar{u} / u)$ or $(\bar{v} u / \bar{u} v \bar{u})$. If it is the former, then the new element $z$ in the following pair is $\bar{u}^{2} v \bar{u}$ or $u^{2} \bar{v}$. Suppose $z=\bar{u}^{2} v \bar{u}$, in which case $0=d(u, \bar{u})<c(\bar{u}, \overline{\bar{u}} v \bar{u})$. Axiom $A_{4}$ implies that $|\bar{u} v \bar{u}|=|v \bar{u}|-|u|$, which is equivalent to $c(\bar{u}, \overline{v \bar{u}})=|u|$. Then $c(\bar{u}, \bar{v})<c(\bar{u}, \overline{v \bar{u}})$ implying by $A_{4}$ that $|v \bar{u}|=2|u|-|\bar{u} v|$. It follows that $|\bar{u} v \bar{u}|=|u|-|\bar{u} v|<0$, a contradiction. It is easily seen that $z \neq$ $u^{2} \bar{v} u$. Thus the second pair following $(u, v)$ must be $(\bar{v} u / \bar{u} v \bar{u})$.

Let $r$ be the integer such that the sequence from $(u, v)$ on has the form

$$
(u, v),(\bar{u} v, u),(\bar{u} v, \bar{u} v \bar{u}),\left(\bar{u} v,(\bar{u} v)^{2} \bar{u}\right), \ldots,\left(\bar{u} v,(\bar{u} v)^{r-1} \bar{u}\right),\left(\bar{u} v /(\bar{u} v)^{r} \bar{u}\right)
$$

and the next term, say the $R$ th term, is not $\left(\bar{u} v /(\bar{u} v)^{r+1} \bar{u}\right)$. The new element $z$ in the $R$ th term is $\bar{u} v u(\bar{v} u)^{r}, \bar{v} u u(\bar{v} u)^{r}$, or $(\bar{u} v)^{r+1} \bar{u}$; it can easily be shown to be $(\bar{u} v)^{r+1} \bar{u}$ or a contradiction results. Thus the $R$ th term is $\left((\bar{u} v)^{r} \bar{u} /(\bar{u} v)^{r+1} \bar{u}\right)$. The new element in the $(R+1)$ th term is $(\bar{u} v)^{r} \bar{u}(\bar{u} v)^{r+1} \bar{u}$ or $(\bar{u} v)^{r+1} \bar{u}(\bar{u} v)^{r} \bar{u}$. But both of these lead to contradictions. Therefore $u$ is not cyclically reduced.

This completes the proof that given any $N$ there exists $n>N$ such that some element of $\left(x_{n}, y_{n}\right)$ is not cyclically reduced.

Lemma 5.5. Let $\left(x_{i}, y_{i}\right)$ be an infinite reduction sequence for $x, y \in G$ where limit $_{i \rightarrow \infty}\left|x_{i}\right|=$ limit $_{i \rightarrow \infty}\left|y_{i}\right|=\alpha>0$. If the elements of $\left(x^{*}, y^{*}\right)$, the Nth pair of the reduction sequence, are not cyclically reduced and bave length less than $2 a$, then for $n \geq N$ neither $x_{n}$ nor $y_{n}$ is cyclically reduced.

Proof. We assume the existence of $n>N$ such that some element of 
$\left(x_{n}, y_{n}\right)$ is cyclically reduced and derive a contradiction. Choose $n$ to be minimal and let $z$ be the element of the $n$th pair that is cyclically reduced. Write $z=x^{\prime} y^{\prime}$ where $\left(x^{\prime}, y^{\prime}\right)$ is the $(n-1)$ th pair in the reduction sequence. It is easy to see that neither $c\left(y^{\prime}, x^{\prime}\right)$ nor $c\left(y^{\prime}, \bar{x}^{\prime}\right)$ is zero. Since $\left|x^{\prime} y^{\prime}\right|<\left|y^{\prime}\right|$, $c\left(x^{\prime}, \bar{y}^{\prime}\right)>1 / 2\left|x^{\prime}\right|$. Using this and the fact that $y^{\prime}$ is not cyclically reduced, $c\left(y^{\prime}, x^{\prime}\right)>0$ by $A_{4}$. And from $c\left(x^{\prime}, \bar{x}^{\prime}\right)>0$ and $c\left(y^{\prime}, x^{\prime}\right)>0$ it follows by $A_{4}$ that $c\left(y^{\prime}, \bar{x}^{\prime}\right)>0$. Also $c\left(x^{\prime} y^{\prime}, \bar{x}^{\prime}\right) \neq 0$. For $0=c\left(x^{\prime} y^{\prime}, \bar{x}^{\prime}\right)<c\left(y^{\prime}, \bar{x}^{\prime}\right)$ implies by $A_{4}$ that $0=c\left(x^{\prime} y^{\prime}, y^{\prime}\right)$ which is equivalent to $\left|x^{\prime} y^{\prime}\right|=\left|x^{\prime}\right|-\left|y^{\prime}\right| \leq 0$. Since $z$ is cyclically reduced, $0=c\left(x^{\prime} y^{\prime}, \overline{x^{\prime} y^{\prime}}\right)<c\left(x^{\prime} y^{\prime}, \bar{x}^{\prime}\right)$. Axiom $A_{4}$ then implies $c\left(\overline{x^{\prime} y^{\prime}}, \bar{x}^{\prime}\right)=0$ so $\left|x^{\prime} y^{\prime}\right|=\left|y^{\prime}\right|-\left|x^{\prime}\right|$. Since $\left|y^{\prime}\right|<2 \alpha$ and $\left|x^{\prime}\right|>\alpha,\left|x^{\prime} y^{\prime}\right|<\alpha$, a contradiction. Therefore no element $z$ in the reduction sequence after $\left(x^{*}, y^{*}\right)$ is cyclically reduced.

The next step is to establish some trivial lemmas that we need in the sequel.

Lemmas 5.6-5.8. Let $\left(x_{i}, y_{i}\right)$ be an infinite reduction sequence for $x, y \in$ G. Assume that the limit numbers for this sequence are both equal to some nonzero real number $a$ and that no group element which occurs in this sequence is cyclically reduced. Let $(a, b)$ be a pair in the reduction sequence and assume that the following pair is $(a / a b)$ where $|a b| \leq \operatorname{minimum}\{|a \bar{b}|,|\bar{a} b|,|\bar{a} \bar{b}|\}$. Let $c=c(a, \bar{a})$ and $d=d(b, \bar{b})$.

Lemma 5.6. The following hold:

(5.6a) If $c=d$, then $c=c(\bar{a}, \bar{b})=c(a, b) \leq c(\bar{a}, b) \leq c(a, \bar{b})$.

(5.6b) If $c<d$, then one of the following occurs:

(i) $c(a, \bar{a})=c(\bar{a}, \bar{b})=c(\bar{a}, b)<c(b, \bar{b})=c(a, b)<c(a, \bar{b})$;

(ii) $\quad c(a, \bar{a})=c(\bar{a}, \bar{b})=c(\bar{a}, b)<c(a, \bar{b})=c(a, b)<c(b, \bar{b})$;

(iii) $\quad c(a, \bar{a})=c(\bar{a}, \bar{b})=c(\bar{a}, b)<c(a, \bar{b})=c(a, b)=c(b, \bar{b})$.

(5.6c) If $d<c$, then $c(b, \bar{b})=c(\bar{a}, b)=c(a, b)<c(a, \bar{a})=c(\bar{a}, \bar{b})<c(a, \bar{b})$.

Lemma 5.7. If $c<d \leq 1 / 2 \alpha$ or $d<c \leq 1 / 2 \alpha$, then $a^{2} b$ is a component of the second pair after $(a, b),|a b|=|b|-|a|+2 c$, and $c(a b, \overline{a b})=d$.

Lemma 5.8. If $d<c$ and $2 d \leq a \leq 2 c$, then $a^{2} b$ occurs in the second pair after $(a, b), c(a b, \overline{a b})=d$, and either $|a b|<|a|$ or $|a b|=|b|-|a|+2 c$.

Proof of 5.6. Since $a b$ occurs in the pair after $(a, b), c(a, a)<1 / 2|a|<$ $c(a, \bar{b})$ so $\mathrm{A}_{4}$ implies $c(a, \bar{a})=c(\bar{a}, \bar{b})$. Also by our choice of $a b, c(a, \bar{b}) \geq$ maximum $\{c(a, b), c(\bar{a}, \bar{b}), c(\bar{a}, b)\}$. These results hold for all three cases.

Assume $c=d$. Then $c(b, \bar{b})<c(a, \bar{b})$ so $A_{4}$ implies $c(b, \bar{b})=c(a, b)$. And from $c(a, \bar{a})=c(a, b)$ we conclude by $\mathrm{A}_{4}$ that $c(\bar{a}, b) \geq c(a, \bar{a})$.

Assume $c<d$. Then $c(\bar{a}, \bar{b})<c(b, \bar{b})$ so $A_{4}$ implies $c(\bar{a}, \bar{b})=c(\bar{a}, b)$. Compare $c(b, \bar{b})$ and $c(a, \bar{b})$. If $c(b, \bar{b})<c(a, \bar{b})$, then $c(b, \bar{b})=c(a, b)$ by $\mathrm{A}_{4}$. 
If $c(a, \bar{b})<c(b, \bar{b})$, then $c(a, \bar{b})=c(a, b)$ by $A_{4}$. Finally suppose $c(a, \bar{b})=$ $c(b, \bar{b})$. Axiom $A_{4}$ implies $c(a, b) \geq c(a, \bar{b})$. But as we noted in the beginning of the proof $c(a, \bar{b}) \geq c(a, b)$. Therefore $c(a, b)=c(a, \bar{b})$.

Assume $d<c$. Then $d=c(b, \bar{b})<c(\bar{a}, \bar{b})=c$, so $A_{4}$ implies $c(b, \bar{b})=$ $c(b, \bar{a})$. And since $c(a, \bar{b})>c(a, \bar{a})>c(b, \bar{b}), c(b, \bar{b})=c(a, b)$ by $A_{4}$.

Proof of 5.7. The arguments for both the case when $c<d \leq 1 / 2 a$ and the case when $d<c \leq 1 / 2 a$ are similar. We will only present the details for $c<d \leq 1 / 2 a$.

Since $c(a, \bar{b})>1 / 2|a|>1 / 2 \alpha$ and $c(b, \bar{b})=d \leq 1 / 2 \alpha$, the results in $(5.6 \mathrm{~b}(\mathrm{i}))$ are applicable here. The possibilities for the new element in the second pair after $(a, b)$ are $a \bar{b} \bar{a}, \bar{a} \bar{b} \bar{a}$, and $a^{2} b$. Suppose first that $a \bar{b} \bar{a}$ occurs, that is $|a \bar{b} \bar{a}|<$ maximum $\{|a|,|a b|\}$. Then $1 / 2 a<c(a, \overline{\overline{b a}})$. Now $c(a, b)=d$ by $(5.6 \mathrm{~b}(\mathrm{i}))$ and $d \leq 1 / 2 a$ by hypothesis, so it follows that $c(a, b)<c(a, \overline{\overline{b a}})$. Axiom $A_{4}$ implies $c(a, b)=c(b, \bar{b} \bar{a})$ so $|a b|=|a|-|b|+2 d$. But then $|a b|<\alpha$, a contradiction. Therefore $|a \bar{b} a| \geq \operatorname{maximum}\{|a|,|a b|\}$. Secondly suppose $\bar{a} \bar{b} \bar{a}$ occurs. Then $1 / 2 a$ $<c(\bar{a}, \overline{\bar{b}} \bar{a})$. Since $c=c(\bar{a}, b)$ by $(5.6 \mathrm{~b}(\mathrm{i}))$ and $c<1 / 2 \alpha, \mathrm{A}_{4}$ implies $c(\bar{a}, b)=$ $c(b, \bar{b} \bar{a})$ so $|a b|=|a|-|b|+2 c$. But then $|a b|<\alpha$, a contradiction. Therefore $a^{2} b$ must occur. Then $1 / 2 a<c(a, \bar{a} \bar{b})$ so $c(a, \bar{a})<c(a, \overline{a b})$. Axiom $\mathrm{A}_{4}$ implies $c(a, \bar{a})=c(\bar{a}, \overline{a b})$ which yields $|a b|=|b|-|a|+2 c$. Note that $|a b|>a$ but $2 c$ $<a$ so $|a|<|b|$.

It remains to prove that $c(a b, \overline{a b})=d$. We first establish that $c(a b, a)=d$. Compare $c(a b, a)$ with $c(b, a)$ which by $(5.6 \mathrm{~b}(\mathrm{i}))$ is $d$. If $c(a, b)<c(a, a b)$, then $A_{4}$ implies $c(a, b)=c(b, a b)$ so $|a b|=|a|-|b|+2 d$. But then $|a b|<a$ which is impossible. If $c(a, a b)<c(a, b)$, then $A_{4}$ implies $c(a, a b)=c(a b, b)$ so $|a b a|=2|a|-|b|$. But since $|a|<|b|$ this contradicts the fact, established earlier in the proof, that $|a b \bar{a}| \geq \operatorname{maximum}\{|a|,|a b|\}$. Since $\left|a^{2} b\right|<$ maximum $\{|a|,|a b|\}, c(a, \overline{a b})>\operatorname{minimum}\{1 / 2|a|, 1 / 2|a b|\}>1 / 2 a \geq d$. Axiom $A_{4}$ then yie lds $c(a b, \overline{a b})=d$.

Proof of 5.8. Since $d<c$, the results of (5.6c) hold. One of $a \bar{b} \bar{a}, \bar{a} \bar{b} \bar{a}$, and $a^{2} b$ must occur in the term following $(a / a b)$. Suppose $a \bar{b} \bar{a}$ occurs. Then $c(a, \bar{b} \bar{a})>\operatorname{minimum}\{1 / 2|a|, 1 / 2|a b|\}>1 / 2 a \geq d$. Since $c(a, b)=d$ by $(5.6 c), A_{4}$ implies $d=c(b, \overline{\bar{b} a})$ so $|a b|=|a|-|b|+2 d$. But then $|a b| \leq \alpha$, a contradiction. Secondly assume $\bar{a} \bar{b} \bar{a}$ occurs. Then $d<c(\bar{a}, \bar{b} \bar{a})$. Since $c(\bar{a}, b)=d$ by (5.6c), $A_{4}$ yields $d=c(b, \bar{b} \bar{a})$ which as seen above implies a contradiction. Therefore $a^{2} b$ must occur.

Now consider $c(a b, \overline{a b})$. Since $|a| \leq|b|$ and $|a b|>a \geq 2 d, c(a b, b)>d$. And $c(a, b)=d$ by $(5.6 \mathrm{c})$. Therefore $\mathrm{A}_{4}$ implies $c(a b, a)=d$. Since $a^{2} b$ occurs in the sequence $c(a, \bar{a} \bar{b})>$ minimum $\{1 / 2|a|, 1 / 2|a b|\}>1 / 2 \alpha \geq d$. An application of $A_{4}$ yields $c(a b, \overline{a b})=d$.

Finally consider $|a b|$. If $c>1 / 2|a b|$, then $|a b|<|a|$. And if $c \leq 1 / 2|a b|$, then 
$c=c(a, \bar{a})<c(a, \overline{a b})$ so $\mathrm{A}_{4}$ implies $c=c(\bar{a}, \overline{a b})$. Thus $|a b|=|b|-|a|+2 c$.

Proposition 5.9. Let $\left(x_{i}, y_{i}\right)$ be an infinite reduction sequence for $x, y \in G$. Assume that the limit numbers for this sequence are both equal to some nonzero number $\alpha$ and that no element which occurs in this sequence is cyclically reduced. Furthermore assume that for all $i,\left(x_{i+1}, y_{i+1}\right)=\left(x_{i} / z\right)$ where $z$ has the shortest length of all the possible choices. Then limit ${ }_{i \rightarrow \infty} c\left(y_{i}, \bar{y}_{i}\right)=1 / 2 \alpha=$ $\operatorname{limit}_{i \rightarrow \infty} c\left(x_{i}, \bar{x}_{i}\right)$.

Proof. Fix $0<\epsilon<1 / 4 a$. Let $\left(a^{\prime}, b^{\prime}\right)$ be a pair in our reduction sequence such that $\left|b^{\prime}\right|-a<\epsilon$. Let $(a, b)$ be an arbitrary pair beyond $\left(a^{\prime}, b^{\prime}\right)$. Without loss of generality assume $a b$ is in the next pair. By our assumption this pair is $(a / a b)$ where $|a b| \leq$ minimum $\{|\bar{a} b|,|\bar{a} \bar{b}|,|a \bar{b}|\}$. For convenience we will refer to the $n$th pair following $(a, b)$ as the $(n+1)$ th pair; thus $(a / a b)$ is the second pair. Let $c=c(a, \bar{a})$ and $d=c(b, \bar{b})$. It suffices to prove that $\alpha-2 \epsilon<2 c<\alpha+\epsilon$ and $\alpha-$ $2 \epsilon<2 d<a+\epsilon$. For the time being assume that $d<1 / 2 \alpha$.

Case 1. $c<d$.

By Lemma 5.7, $2 c=|a b|+|a|-|b|$. Since $|a b|>a,|a|>\alpha$, and $|b|<a$ $+\epsilon, 2 c>\alpha-\epsilon$. Hence $\alpha>2 d>2 c>\alpha-\epsilon$.

Case 2. $d<c<1 / 2 \alpha$.

By Lemma 5.7, $c(a b, \overline{a b})=d$. If $|a b| \leq|a|$, then the second pair is $(a b, a)$ with $c(a b, \overline{a b})<c(a, \bar{a})<1 / 2 a$. Thus we are in Case 1 and hence done. If $|a|<$ $|a b|$, then consider the third pair $\left(a / b_{3}\right)$. By Lemma 5.7, $b_{3}=a a b$ and $c\left(b_{3}, \bar{b}_{3}\right)$ $=d$. If $|a a b|<|a|$, we are in Case 1. Otherwise consider the fourth pair $\left(a / b_{4}\right)$. Since Proposition 4.7 implies ' $a$ ' becomes a ' $b_{i}$ ', we can conclude by Case 1 that $2 c$ and $2 d$ are within $2 \epsilon$ of $\alpha$.

Case 3. $c=d$.

(3.1) $a^{2} b$ occurs in the third pair.

Then $c(a, \bar{a})<c(a, \overline{a b})$. Axiom $A_{4}$ implies $c(a, \bar{a})=c(\bar{a}, \overline{a b})$, so that $2 c=$ $|a|+|a b|-|b|$. Since $|a|>\alpha,|a b|>\alpha$, and $|b|<\alpha+\epsilon$, it follows that $2 c>a$ $-\epsilon$.

(3.2) $a \bar{b} \bar{a}$ occurs in the third pair.

Then $c(a, \bar{a})<c(a, \overline{\bar{b} a})$. Since (5.6a) implies $c(a, b)=c, c(a, b)=c(b, \overline{\bar{b}} \bar{a})$ by $A_{4}$ so $2 c=|b|+|a b|-|a|$. This is a contradiction; $2 c<a$ but $|b|+|a b|-|a|$ $\geq|a b|>a$.

(3.3) $a b a$ occurs in the third pair.

Then $c(a b, \bar{a})>c(a, \bar{a})$, so $c(a b, a)=c$. First assume $|a|<|a b|$. Then the third pair is $(a / a b a)$, so a coordinate of the fourth pair is $a b a^{2}$ or $a^{2} b a$. If it is $a b a^{2}$, then $c(a, \bar{a})<c(a b a, \bar{a})$. Axiom $\mathrm{A}_{4}$ implies $c=c(a b a, a)$, so that $2 c=$ $|a b a|+|a|-|a b|>a-\epsilon$. Suppose $a^{2} b a$ occurs in the fourth pair. Then $c(a, \bar{a})$ 
$<c(a, \overline{a b a})$, so $\mathrm{A}_{4}$ implies $c=c(\bar{a}, \overline{a b a})$. Hence $2 c=|a|+|a b a|-|b a|$. We need to consider $|b a|$. Let $M=c(a, \ddot{b})+c(b, \bar{a})$. Since $c(b, \bar{b}) \leq c(\bar{a}, b) \leq c(a, \bar{b})$ by (5.6a), $M \geq|b|$ implies $c(a, \bar{b}) \geq 1 / 2|b|$. But then $|a b| \leq|a|$, contrary to our assumption. Therefore $M<|b|$ so $c(a b, \bar{a})=c(b, \bar{a})$ by Proposition 2.4. Since $|a b a|<|a b|, c(a b, \bar{a})>1 / 2|a|$. Thus $c(b, \bar{a})>1 / 2|a|$, so $|b a|<|b|$. Then $2 c>|a|+$ $|a b a|-|b|>a-\epsilon$.

Secondly assume $|a b| \leq|a|$. Then the second pair is $(a b, a)$ and the third pair is $(a b a / a b)$. Since $\left|(a b)^{n} a\right| \geq\left|(a b)^{n}\right|-|a|$ by Proposition 2.1 and the lengths $\left|(a b)^{n}\right|$ are unbounded by Proposition 2.6, the lengths $\left|(a b)^{n} a\right|$ are unbounded. Let $m$ be the positive integer such that the sequence from $(a, b)$ is as follows: $(a b, a),\left(a b,(a b)^{1} a\right),\left(a b,(a b)^{2} a\right),\left(a b,(a b)^{3} a\right), \ldots,\left(a b,(a b)^{m-1} a\right),\left(a b /(a b)^{m} a\right)$ and the next term, say the $R$ th term, is not $\left(a b /(a b)^{m+1} a\right)$. The new element $z$ in the Rth pair is $(a b)^{m} a \bar{b} \bar{a},(a b)^{m} a a b$, or $(a b)^{m+1} a$. Suppose $z=(a b)^{m} a \bar{b} \bar{a}$ in which case $c\left((a b)^{m} a, a b\right)>c(a, \bar{a})=c(a b, a)$. Let $t$ be the first integer such that $c\left((a b)^{t} a, a b\right)>c(a, \bar{a})$ and note that $0<t \leq m$. Since $c\left((a b)^{t} a, a b\right)>c(a, \bar{a})$ $\geq c\left((a b)^{t-1} a, a b\right), A_{4}$ implies $c\left((a b)^{t} a,(a b)^{t-1} a\right) \leq c$. Hence $\left|(a b)^{t} a\right|+\left|(a b)^{t-1} a\right|$ $-|a b| \leq 2 c$. Since $\left|(a b)^{t} a\right|>\alpha,\left|(a b)^{t-1} a\right|>a$, and $|a b|<\alpha+\epsilon, 2 c \geq a-\epsilon$ as desired. Suppose $z=(a b)^{m} a a b$ in which case $c\left((a b)^{m} a, \overline{a b}\right)>1 / 2 a>c(a, \bar{a})$. If $c(a, \bar{a})<c(\bar{b} \bar{a}, a)$, then the argument in Case 3 (3.1) yields $2 c>a-\epsilon$. Assume then that $c(\bar{b} \bar{a}, a) \leq c(a, \bar{a})$. Let $t$ be the first positive integer such that $c\left((a b)^{t} a, \overline{a b}\right)$ $>c(a, \bar{a})$ and note that $0<t \leq m$. Since $c\left((a b)^{t-1} a, \overline{a b}\right)<c\left((a b)^{t} a, \overline{a b}\right), \mathrm{A}_{4}$ implies $c\left((a b)^{t} a,(a b)^{t-1} a\right) \leq c(a, \bar{a})=c$. As above this yields our desired result, $2 c \geq a-\epsilon$. Finally suppose $z=(a b)^{m+1} a$. Then the Rth pair is $\left((a b)^{m} a /(a b)^{m+1} a\right)$. The new coordinate of the $(R+1)$ th pair is $(a b)^{m} a(a b)^{m+1} a$ or $(a b)^{m+1} a(a b)^{m} a$. Since the arguments are similar for both of these, we will only present the details of the former. Assume that $(a b)^{m} a(a b)^{m+1} a$ occurs in the $(R+1)$ th term. Consider $c\left((a, b)^{m+1} a,(a b)^{m+1} a\right)$. Suppose first that it is less than or equal to $c(a, \bar{a})$. Since $(a b)^{m} a(a b)^{m+1} a$ occurs in the sequence, $c\left((a b)^{m} a, \overline{\left.(a b)^{m+1} a\right)}>\right.$ $c(a, \bar{a})$. By $\mathrm{A}_{4}, c\left((a b)^{m+1} a, \overline{\left.(a b)^{m+1} a\right)}=c\left((a b)^{m+1} a,(a b)^{m} a\right) \leq 2 c\right.$. Thus $2 c \geq$ $\left|(a b)^{m+1} a\right|+\left|(a b)^{m} a\right|-|a b|>\alpha-\epsilon$. Secondly suppose $c\left((a b)^{m+1} a, \overline{\left.(a b)^{m+1} a\right)}>\right.$ $c(a, \bar{a})$. Then let $t$ be the smallest integer such that $c\left((a b)^{t} a,(\overline{a b})^{t} a\right)>c(a, \bar{a})$. Note that $1 \leq t \leq m+1$. Let $T=c\left((a b)^{t} a,\left(\overline{a b)^{t}-T_{a}}\right)\right.$. If $T>c$, then since $c\left((a b)^{t-1} a,(\overline{a b})^{\bar{t}-1} a\right) \leq c<T, A_{4}$ implies that $c\left((a b)^{t-1} a, \overline{(a b)^{t-1} a}\right)=$ $c\left((a b)^{t-1} a,(a b)^{t} a\right)$. Hence $2 c \geq\left|(a b)^{t-1} a\right|+\left|(a b)^{t} a\right|-|a b|>a-\epsilon$ as desired.

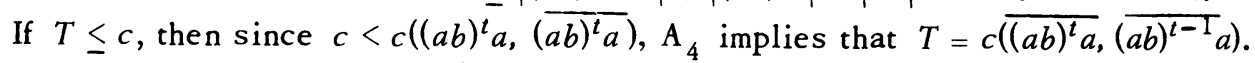
Hence $2 c \geq\left|(a b)^{t} a\right|+\left|(a b)^{t-1} a\right|-|\bar{a} \bar{b}|$. If $|b a|<a+2 \epsilon$, then $2 c>a-2 \epsilon$, and we have our result. We claim that this is always the case. Compare $c(\bar{a}, b)$ and $c(\bar{a}, a b)$. If $c(\bar{a}, b)<c(\bar{a}, a b)$, then $\mathrm{A}_{4}$ implies $c(\bar{a}, b)=c(b, a b)$. Since $c(b, a b)$ 
$\geq 1 / 2|a b|$, it follows that $|\bar{a} \bar{b}| \leq|a|+|b|-|a b|<\alpha+2 \epsilon$. If $c(\bar{a}, a b) \leq c(\bar{a}, b)$, then $|\bar{a} \bar{b}| \leq|b|+|a b a|-|a b|<a+2 \epsilon$.

Case 4. $d<1 / 2 \alpha \leq c$.

By Proposition 4.7 our sequence has the form $(a, b),\left(a, b_{1}\right),\left(a, b_{2}\right), \ldots$, $\left(a, b_{n}\right),\left(a^{\prime}, a\right)$. Let $a=b^{\prime}$. By Lemma 5.7 if $2 c=\alpha$ and by Lemma 5.8 if $2 c>a$, we see that $c\left(b_{i}, \overline{b_{i}}\right)=d=c\left(a^{\prime}, \bar{a}^{\prime}\right)$. Assume the pair following $\left(a^{\prime}, b^{\prime}\right)$ is $\left(a^{\prime} / a^{\prime} b^{\prime}\right)$. Compare $c\left(a^{\prime}, \overline{a^{\prime}}\right)=d$ and $c\left(a^{\prime}, \overline{a^{\prime} b^{\prime}}\right)$. If $c\left(a^{\prime}, \overline{a^{\prime}}\right)<c\left(a^{\prime}, \overline{a^{\prime} b^{\prime}}\right)$, then $\mathrm{A}_{4}$ implies $c\left(a^{\prime}, \overline{a^{\prime}}\right)=c\left(\overline{a^{\prime}}, \overline{a^{\prime} b^{\prime}}\right)$. Therefore $2 d=\left|a^{\prime}\right|+\left|a^{\prime} b^{\prime}\right|-\left|b^{\prime}\right|>a-\epsilon$ and we are done. If $c\left(a^{\prime}, \overline{a^{\prime} b^{\prime}}\right)<c\left(a^{\prime}, \overline{a^{\prime}}\right)$, then $c\left(a^{\prime}, \overline{a^{\prime} b^{\prime}}\right)=c\left(\overline{a^{\prime}}, \overline{a^{\prime} b^{\prime}}\right)$ so $\left|a^{\prime} a^{\prime} b^{\prime}\right|=$ $\left|b^{\prime}\right|$. Since $c\left(\overline{a^{\prime}}, \overline{a^{\prime} b^{\prime}}\right)<d, 2 d>\left|a^{\prime}\right|+\left|a^{\prime} b^{\prime}\right|-\left|b^{\prime}\right|>a-\epsilon$. Suppose $c\left(a^{\prime}, \overline{a^{\prime}}\right)=$ $c\left(a^{\prime}, \overline{a^{\prime} b^{\prime}}\right)$. Then $\left|a^{\prime} a^{\prime} b^{\prime}\right|>\operatorname{maximum}\left\{\left|a^{\prime}\right|,\left|a^{\prime} b^{\prime}\right|\right\}$, so $a^{\prime} a^{\prime} b$ is not a term in the pair following $\left(a^{\prime} / a^{\prime} b^{\prime}\right)$. Hence the new element in this pair is either $a^{\prime} b^{\prime} a^{\prime}$ or $a^{\prime} b^{\prime} \overline{a^{\prime}}$. If the latter, $c\left(a^{\prime} b^{\prime}, a^{\prime}\right)>d$. Axiom $A_{4}$ implies $c\left(a^{\prime} b^{\prime}, \overline{a^{\prime} b^{\prime}}\right)=d$. Then $\left(a^{\prime} / a^{\prime} b^{\prime}\right)$ is a pair such that $d=c\left(a^{\prime}, \overline{a^{\prime}}\right)=c\left(a^{\prime} b^{\prime}, \overline{a^{\prime} b^{\prime}}\right)<1 / 2 a$ and we are done by Case 3. Assume $a^{\prime} b^{\prime} a^{\prime}$ occurs in the pair following $\left(a^{\prime} / a^{\prime} b^{\prime}\right)$. Consider $c\left(a^{\prime}, \overline{b^{\prime}}\right)+c\left(b^{\prime}, \overline{a^{\prime}}\right)$. Since $c\left(a^{\prime}, \overline{b^{\prime}}\right)>1 / 2 a$ and $c\left(b^{\prime}, \overline{b^{\prime}}\right) \geq 1 / 2 a, A_{4}$ implies $c\left(a^{\prime}, b^{\prime}\right) \geq 1 / 2 a$. This together with $c\left(a^{\prime}, \overline{a^{\prime}}\right)=d<1 / 2 a$ yields $c\left(\overline{a^{\prime}}, b^{\prime}\right)=d$. Now if $c\left(a^{\prime}, \overline{b^{\prime}}\right)<\left|b^{\prime}\right|-d$, then Proposition 2.4 implies $c\left(a^{\prime} b^{\prime}, \overline{a^{\prime}}\right)=c\left(b^{\prime}, \overline{a^{\prime}}\right)=d<$ $1 / 2 a$, contrary to the fact that $\left|a^{\prime} b^{\prime} a^{\prime}\right|<\operatorname{maximum}\left\{\left|a^{\prime} b^{\prime}\right|,\left|a^{\prime}\right|\right\}$. And if $c\left(a^{\prime}, \overline{b^{\prime}}\right) \geq$ $\left|b^{\prime}\right|-d$, then $2 d \geq\left|b^{\prime}\right|-\left|a^{\prime}\right|+\left|a^{\prime} b^{\prime}\right| \geq\left|a^{\prime} b^{\prime}\right|>a$, a contradiction.

Thus if $d<1 / 2 \alpha$, then both $c$ and $d$ are within a couple of $\epsilon$ 's of $1 / 2 a$. Now assume $d \geq 1 / 2 \alpha$. Then $\alpha \leq 2 d<|b|<\alpha+\epsilon$. If $c \geq 1 / 2 \alpha$, then $\alpha \leq 2 c<|a|<a+\epsilon$ and we are done. Assume $c(a, \bar{a})=c<1 / 2 a$. Since Proposition 4.7 implies every ' $a$ ' becomes a ' $b$ ', $\alpha-2 \epsilon<2 c$ by the above arguments. This completes the proof that limit ${ }_{i \rightarrow \infty} c\left(y_{i}, \overline{y_{i}}\right)=\operatorname{limit}_{i \rightarrow \infty} c\left(x_{i}, \overline{x_{i}}\right)=1 / 2 \alpha$.

In the proof that $\langle x, y\rangle$, is abelian we need a proposition concerning the length of a product of elements.

Proposition 5.10. If $a_{i} \in G$ such that $\left|a_{i}\right|>2 d$ and $c\left(a_{i}, \overline{a_{i+1}}\right) \geq d$ for $1 \leq$ $i<n$, then

$$
\left|a_{1} a_{2} \cdots a_{n}\right| \leq 2 d+n \underset{1 \leq i \leq n}{\operatorname{maximum}}\left\{\left|a_{i}\right|-2 d\right\}
$$

Proof. We will prove this by induction on $n$. The result is obvious for $n=$ 1. Assume it is true for $n-1$ elements where $n>1$. If $c\left(a_{1} a_{2} \cdots a_{n-1}, \bar{a}_{n}\right)$ $\geq d$, then $\left|a_{1} a_{2} \cdots a_{n}\right| \leq\left|a_{1} a_{2} \cdots a_{n-1}\right|+\left|a_{n}\right|-2 d$. By the induction hypothes is applied to $\left|a_{1} a_{2} \cdots a_{n-1}\right|$,

$$
\begin{aligned}
\left|a_{1} a_{2} \cdots a_{n}\right| & \leq 2 d+(n-1) \operatorname{maximum}_{1 \leq i \leq n-1}\left\{\left|a_{i}\right|-2 d\right\}+\left|a_{n}\right|-2 d \\
& \leq 2 d+n \underset{1 \leq i \leq n}{\operatorname{maximum}}\left\{\left|a_{i}\right|-2 d\right\} .
\end{aligned}
$$


If $c\left(a_{1} a_{2} \cdots a_{n-1}, \overrightarrow{a_{n}}\right)<d$, then $A_{4}$ implies $c\left(a_{1} a_{2} \cdots a_{n-1}, \overrightarrow{a_{n}}=\right.$ $c\left(a_{1} a_{2} \cdots a_{n-1}, a_{n-1}\right)$ since by hypothesis $c\left(a_{n-1}, \overline{a_{n}}\right) \geq d$. Thus $\mid a_{1} a_{2} \cdots$ $a_{n-1} a_{n}|=| a_{1} a_{2} \cdots a_{n-2}|+| a_{n}|-| a_{n-1} \mid$. By the induction hypothesis applied to $\left|a_{1} a_{2} \cdots a_{n-2}\right|$,

$$
\begin{aligned}
\left|a_{1} a_{2} \cdots a_{n}\right| & \leq 2 d+(n-2) \operatorname{maximum}_{1 \leq i \leq n-2}\left\{\left|a_{i}\right|-2 d\right\}+\left(\left|a_{n}\right|-2 d\right)+\left(2 d-\left|a_{n-1}\right|\right) \\
& \leq 2 d+n \operatorname{maximum}_{1 \leq i \leq n}\left\{\left|a_{i}\right|-2 d\right\} .
\end{aligned}
$$

Proposition 5.11. If every reduction sequence for $x, y \in G$ is infinite with limit numbers that are equal but not zero, then $\langle x, y\rangle$ is abelian.

Proof. Let $\left(x_{n}, y_{n}\right)$ be an infinite reduction sequence for $x, y \in G$ with limit numbers $\alpha=\beta>0$. Assume for all $i,\left(x_{i+1}, y_{i+1}\right)=\left(x_{i} / z\right)$ where $z$ has the shortest length of all the possible choices. In view of Lemmas 5.4 and 5.5 no generality is lost in assuming that no element which occurs in this sequence is cyclically reduced. For each $n$, let

$$
\Gamma_{n}=\left\{\left[x_{n}, y_{n}\right],\left[x_{n}, \bar{y}_{n}\right],\left[\bar{x}_{n}, y_{n}\right],\left[\bar{x}_{n}, \bar{y}_{n}\right]\right\}
$$

where $[g, b]=\bar{g} \bar{h} g h$. Fix $\epsilon>0$. First we prove that either $x$ and $y$ commute or else for an infinite number of $n$ there exists $K_{n} \in \Gamma_{n}$ such that $\left|K_{n}\right|>\alpha-\epsilon$. Define $c_{n}=c\left(x_{n}, \bar{x}_{n}\right)$ and $d_{n}=c\left(y_{n}, \bar{y}_{n}\right)$. Assume for now that $c_{n}=d_{n}$ for an infinite number of $n$. Let $(a, b)$ be a pair with $c(a, \bar{a})=c(b, \bar{b})$ and $|b|<a+\epsilon$. Assume $(a / a b)$ is the next pair. If $c(\bar{b} a, \overline{b \bar{a}}) \leq c(\bar{b} a, \bar{b})$, then $|\bar{b} a b \bar{a}| \geq|b \bar{a}|+$ $|\bar{b} a b|-|b|$. Since $|b \bar{a}|>\alpha,|\bar{b} a b|>a$, and $a<|b|<\alpha+\epsilon,|\bar{b} a b \bar{a}|>\alpha-\epsilon$. Now assume $c(\bar{b} a, \bar{b})<c(\bar{b} a, \bar{b} \bar{a})$. Then $A_{4}$ implies $c(\bar{b} a, \bar{b})=c(\bar{b}, \overline{b \bar{a}})$. Since $c(\bar{a}, \bar{b})$ $=c(a, b)$ by (5.6a), $|\bar{a} b|=|a \bar{b}|$. It follows that $|\bar{b} a b|=|a|$. Now if $c(\bar{b} a b, a) \leq$ $1 / 2|a|$, then $|\bar{b} a b \bar{a}| \geq|\bar{b} a b|>\alpha$; and if $c(\bar{b} \bar{a} b, \bar{a}) \leq 1 / 2|a|$, then $|\bar{b} \bar{a} b a|>a$. Suppose both are greater than $1 / 2|a|$; then Proposition 2.5 implies $\bar{b} a b=a$ so $a$ and $b$ commute. Since $\langle x, y\rangle=\langle a, b\rangle, x$ and $y$ also commute.

Now assume that there are only finitely many pairs $\left(x_{n}, y_{n}\right)$ with $c_{n}=d_{n}$. Let $(a, b)$ be a pair beyond these such that $|b|<a+\epsilon$. Assume that the next pair is $(a / a b)$. If $c(a b, b a) \leq c(a b, a)$, then $|a b \bar{a} \bar{b}| \geq|\bar{a} \bar{b}|+|a b \bar{a}|-|a|>\alpha-\epsilon$ as $|\bar{a} \bar{b}|>\alpha,|a b \bar{a}|>a$, and $|\bar{a}|<\alpha+\epsilon$. If $c(a \bar{b}, \bar{b} a) \leq c(a \bar{b}, a)$, then $|a \bar{b} \bar{a} b|>$ $a-\epsilon$. Now assume neither of these occur, so $c(a b, a)<c(a b, b a)$ and $c(a \bar{b}, a)$ $<c(a \bar{b}, \bar{b} a)$. From the former $c(a b, a)=c(a, b a)$ by $A_{4}$, so $|a b \bar{a}|=|a b|-|b a|+$ $|b|$. And from the latter $c(a \bar{b}, a)=c(a, \bar{b} a)$ by $A_{4}$ so $|a \bar{b} \bar{a}|=|a \bar{b}|-|\bar{a} b|+|b|$. Combining these we get that $|a b|=|a \bar{b}|-|\bar{a} b|+|b a|$. If $c(a, \bar{a})>c(b, \bar{b})$, then (5.6c) implies $|a b|=|a|+|b|+2 c-4 d$. But since $d<c<1 / 2|a|$, it follows that $|a b|>|b|$, a contradiction. Therefore $c(a, \bar{a})<c(b, \bar{b})$ in this situation. Since $|\bar{a} b|=|\bar{a} \bar{b}|$ by (5.6b), $|a b|=|a \bar{b}|$. It follows that $(5.6 \mathrm{~b}(\mathrm{ii}))$ or $(5.6 \mathrm{~b}(\mathrm{iii}))$ must occur. 
In both of these $c(a, \bar{b}) \leq d$, so $|a b|>|a|$. It is easy to see that given $M$ there is always a pair $(a, b)$ beyond $\left(x_{M}, y_{M}\right)$ for which either $c(a b, b a) \leq c(a b, a)$ or $c(a \bar{b}, \bar{b} a) \leq c(a \bar{b}, a)$ where $(a / a b)$ is the pair following $(a, b)$. For if not, there exists $M$ such that for all pairs $\left(x_{n}, y_{n}\right)$ with $n \geq M,\left|y_{n+1}\right|>\left|x_{n}\right|$. The sequence from $\left(x_{M}, y_{M}\right)$ on has the form $\left(a, y_{M}\right),\left(a, y_{M+1}\right),\left(a, y_{M+2}\right), \ldots$, where $a=x_{M}$. But this contradicts Proposition 4.7. Therefore either $x$ and $y$ commute or else there are an infinite number of pairs $\left(x_{n}, y_{n}\right)$ in our reduction sequence such that $\left|K_{n}\right|>\alpha-\epsilon$ for some $K_{n} \in \Gamma_{n}$.

Suppose for an infinite number of $n$ there is some $K_{n} \in \Gamma_{n}$ such that $\left|K_{n}\right|$ $>a-\epsilon$. As we previously noted, when a Nielsen transformation is applied to $(g, b)$ to obtain $\left(g^{\prime}, b^{\prime}\right)$ there is a one-to-one correspondence between $\Gamma(g, b)$ and $\Gamma\left(g^{\prime}, b^{\prime}\right)$ such that corresponding elements are conjugate. Therefore we can select a subsequence of the se $K_{i}$ such that all elements in it are conjugate. Consider such a sequence $L_{1}, L_{2}, \ldots$ Applying Proposition 5.10 to $\left|L_{i}^{2}\right|$, we get $\left|L_{i}^{2}\right| \leq 2 c_{i}^{\prime}+8\left(\left|b_{i}\right|-2 c_{i}^{\prime}\right)$ where $c_{i}^{\prime}=\operatorname{minimum}\left\{c\left(a_{i}, \bar{a}_{i}\right), c\left(b_{i}, \bar{b}_{i}\right)\right\}$. Since $\left|L_{i}\right|$ $>\alpha-\epsilon$,

$$
\left|L_{i}^{2}\right|-\left|L_{i}\right|<2 c_{i}^{\prime}+8\left(\left|b_{i}\right|-2 c_{i}^{\prime}\right)-\alpha+\epsilon
$$

By Proposition 5.9 as $i \rightarrow \infty, 2 c_{i}^{\prime} \rightarrow \alpha$. And since the limit numbers for our sequence are $a=\beta,\left|b_{i}\right| \rightarrow \alpha$ as $i \rightarrow \infty$. Thus we can make $\left|L_{i}^{2}\right|-\left|L_{i}\right|$ as small as we want by choosing $i$ large and $\epsilon$ small. But $\left|L_{i}^{2}\right|-\left|L_{i}\right|$ is some fixed number $L$ by Theorem 2.8 since all the $L_{i}$ are conjugate. Therefore $L=0$. Axiom $A_{1}$ implies $L_{i}=1$ so $x$ and $y$ commute. This completes the proof of the case $\alpha=\beta>0$. Note that if $\langle x, y\rangle$ is abelian, then for any reduction sequence $\left(x_{i}, y_{i}\right)$ Proposition 2.5 forces $c\left(x_{i}, \bar{x}_{i}\right)=c\left(y_{j}, \bar{y}_{j}\right)=c(x, \bar{x})$.

In summary there are three things that can happen with a reduction sequence $\left(x_{n}, y_{n}\right)$ associated with an arbitrary pair of elements $x, y \in G$. It stops in a finite number of steps and we get a free group of rank 0,1 , or 2 ; it continues indefinitely with $\operatorname{limit}_{n \rightarrow \infty}\left|x_{n}\right|=\operatorname{limit}_{n \rightarrow \infty}\left|y_{n}\right|=0$, in which case $x$ and $y$ are cyclically reduced and commute; or it continues indefinitely with limit ${ }_{n \rightarrow \infty}\left|x_{n}\right|=$ limit $_{n \rightarrow \infty}\left|y_{n}\right|=\alpha>0$, in which case $c\left(x_{n}, \bar{x}_{n}\right)=c\left(y_{n}, \bar{y}_{n}\right)=1 / 2 \alpha$ for all $n$, and $x$ and $y$ commute. Thus an arbitrary pair of elements from $G$ generates either a free or an abelian subgroup.

Theorem 5.12. Let $G$ be a group with a real length function. Then $\langle x, y\rangle$ where $x, y \in G$ is either free or abelian. And if $\langle x, y\rangle$ is free, then $\{x, y\}$ can be fully Nielsen reduced in a finite number of steps.

There are some results of this theorem that are worth mentioning. First note that Proposition 3.7, without adjusting the length function for cyclic reduction, can be stated as follows: 
Proposition 3.7. Let $G$ be a group with a real length function. If $a, b, c$ are nontrivial elements of a commutative subgroup $H$ of $G$, then

$$
\operatorname{maximum}\{c(a, b), c(a, \bar{b})\}=\operatorname{minimum}\{|a|-d,|b|-d\}
$$

and

$$
\operatorname{minimum}\{c(a, b), c(a, \bar{b})\}=d
$$

where $d=c(c, \bar{c})$.

Proposition 5.13. Let $G$ be a group with a real length function. If $x, y^{\prime}, z^{\prime}$ are nontrivial elements of $G$ such that $x$ and $y^{\prime}$ commute and $x$ and $z^{\prime}$ commute, then $y^{\prime}$ and $z^{\prime}$ commute.

Proof. We assume that $y^{\prime}$ and $z^{\prime}$ do not commute and derive a contradiction. By Theorem 5.12 we can fully Nielsen reduce $\left\{y^{\prime}, z^{\prime}\right\}$ to $\{y, z\}$. Since $x$ commutes with both $y^{\prime}$ and $z^{\prime}$ and $\left\langle y^{\prime}, z^{\prime}\right\rangle=\langle y, z\rangle$, it follows that $x$ must commute with both $y$ and $z$. Let $M_{1}$ be the maximal abelian subgroup containing $x$ and $y$, and $M_{2}$ the maximal abelian subgroup containing $x$ and $z$. Since $x \in M_{1} \cap M_{2}, x^{n} \in$ $M_{1} \cap M_{2}$ where $n$ is any integer. In view of Proposition 2.6 we can choose $n$ such that $\left|x^{n}\right|>|y|$ and $\left|x^{n}\right|>|z|$. Let $d=c(x, \bar{x})$. Proposition 3.7 implies maximum $\left\{c\left(x^{n}, y\right), c\left(x^{n}, \bar{y}\right)\right\}$ equals $|y|-d$; for definiteness say $c\left(x^{n}, y\right)=|y|-d$. Similarly maximum $\left\{c\left(x^{n}, z\right), c\left(x^{n}, \bar{z}\right)\right\}=|z|-d$; for definiteness say $c\left(x^{n}, z\right)=$ $|z|-d$. Now $\{y, z\}$ is Nielsen reduced, so $c(y, z) \leq \operatorname{minimum}\{1 / 2|y|, 1 / 2|z|\}$. It follows that $c(y, z)<c\left(y, x^{n}\right)$ since $|y|-d>1 / 2|y|$ by Lemma 3.2 applied to $M_{1}$. Axiom $\mathrm{A}_{4}$ then implies $c(y, z)=c\left(z, x^{n}\right)$. Now $c\left(z, x^{n}\right)>1 / 2|z|$ by Lemma 3.2 applied to $M_{2}$. But $c(y, z) \leq 1 / 2|z|$. Thus we have our contradiction.

This proposition is equivalent to the fact that the centralizer of a nontrivial element is a maximal abelian subgroup.

Corollary 5.14. If $M_{1}$ and $M_{2}$ are distinct maximal abelian subgroups of a group $G$ with a real length function, then $M_{1} \cap M_{2}=\{1\}$.

Proof. Suppose $M_{1} \cap M_{2} \neq\{1\}$. Then there exists $x \in M_{1} \cap M_{2}$ such that $x \neq 1$. Let $y \in M_{1}-\left(M_{1} \cap M_{2}\right)$. There exists some $z \in M_{2}$ such that $y$ and $z$ do not commute. For if $y$ commuted with every element of $M_{2}$, then the subgroup of $G$ generated by $M_{2}$ and $y$ would be an abelian group containing $M_{2}$. But this contradicts the maximality of $M_{2}$. Now $x$ and $y$ commute, $x$ and $z$ commute, but $y$ and $z$ do not commute. By Proposition 5.13 we know that this is impossible. Therefore $M_{1} \cap M_{2}=\{1\}$.

Actually this corollary is equivalent to Proposition 5.13. Assume that two maximal abelian subgroups of $G$ are equal or have trivial intersection. And suppose $x, y$, and $z$ are nontrivial elements of $G$ such that $x y=y x$ and $x z=z x$. 
Let $M_{1}$ be the maximal abelian subgroup containing $x$ and $y$ and $M_{2}$ that containing $x$ and $z$. Since $1 \neq x \in M_{1} \cap M_{2}, M_{1}=M_{2}$. Therefore $y$ and $z$ commute.

There are some more immediate consequences of Proposition 5.13.

Corollary 5.15. If $G$ is a group with a real length function, then $G$ is the disjoint union of its maximal abelian subgroups. (Disjoint is used bere to mean that no pair of subgroups has nontrivial elements in common.)

Corollary 5.16. If $G$ is a group with a real length function, then $G$ is abelian or has trivial center.

Proof. Suppose $G$ is not abelian. Let $x, y \in G$ such that $x y \neq y x$. Define $M_{z}$ for $z \in G$ to be the maximal abelian subgroup containing $C$ and $\{z\}$ where $C$ is the center. Then $M_{x} \neq M_{y}$ but $M_{x} \cap M_{y} \supset C$. Therefore Corollary $5.14 \mathrm{im}$ plies $C=\{1\}$.

\section{REFERENCES}

1. Roger C. Lyndon, Length functions in groups, Math. Scand. 12 (1963), 209-234. MR $29 \# 1246$.

2. W. Magnus, A. Karrass and D. Solitar, Combinatorial group theory: Presentations of groups in terms of generators and relations, Pure and Appl. Math., vol. 13, Interscience, New York, 1966. MR 34 \#7617.

DEP ARTMENT OF MATHEMATICS, BROOKLYN COLLEGE (CUNY), BROOKLYN, NEW YORK 11210 